\title{
Metal ion induced heterogeneity in RNA folding studied by SMFRET
}

With our best wishes to Henryk Kozlowski at the occasion of his anniversary

Richard Boerner†*, Danny Kowerko†, Helena Guiset Miserachs, Michelle F. Schaffer, and Roland K. O. Sigel*

Department of Chemistry B, University of Zurich, Winterthurerstrasse 190, 8057 Zurich, Switzerland.

$\dagger$ Contributed equally to this work.

* Correspondence may be directed to: roland.sigel@chem.uzh.ch, richard.boerner@chem.uzh.ch 


\section{Graphical abstract:}
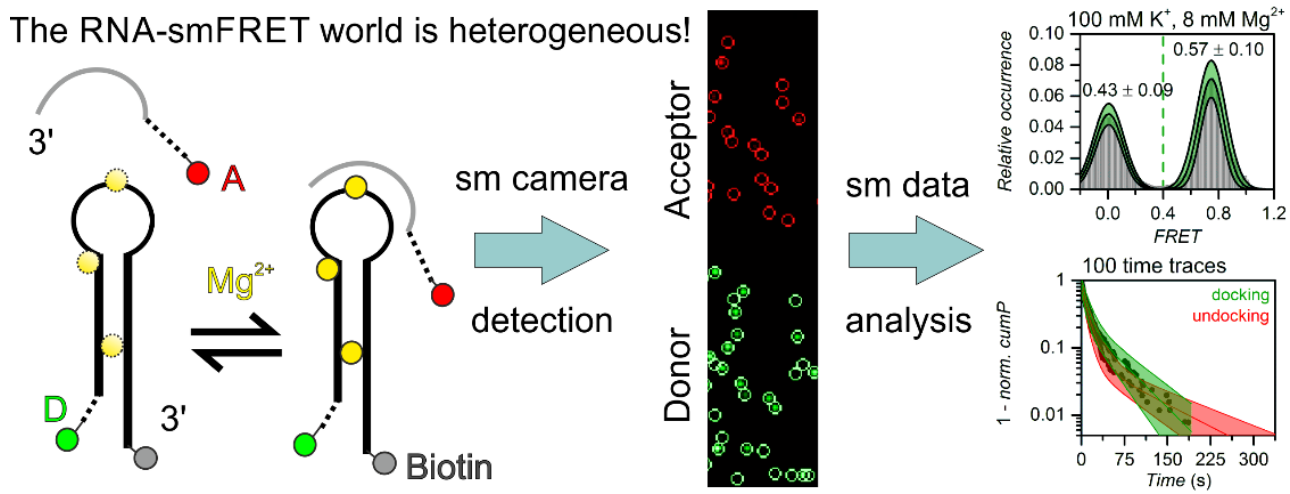

sm data
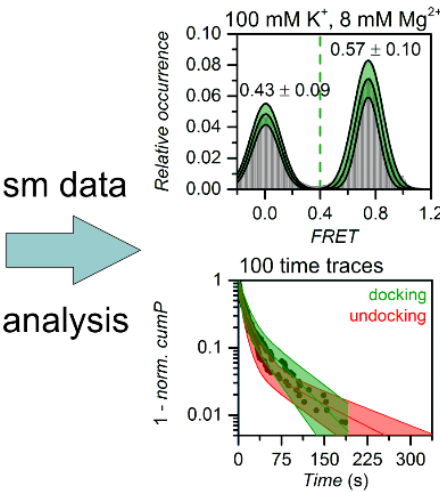

Abbreviations used in the manuscript: $\operatorname{smFRET}{ }^{1}, \mathrm{ALEX}^{2}, \mathrm{~A}^{3}, \mathrm{D}^{4}, \mathrm{PIE}^{5}, \mathrm{ncRNA}^{6}, \mathrm{DNA}^{7}, \mathrm{CD}^{8}$, $\mathrm{ITC}^{9}, \operatorname{TIRF}(\mathrm{M})^{10}, \mathrm{GQ}^{11}, \mathrm{KC}^{12}, \mathrm{ED}^{13}, \mathrm{AV}^{14},(4) \mathrm{WJ}^{15}, \mathrm{wt}^{16}, \mathrm{TDP}^{17}, \mathrm{TCSPC}^{18}, \mathrm{AP}^{19}, \mathrm{nt}^{20}, \mathrm{~L}^{21}$, $\mathrm{EMCCD}^{22}, \mathrm{FWHM}^{23}, \mathrm{BIC}^{24}, \mathrm{D} 135 \mathrm{~L} 14^{25}, \mathrm{EBS}^{26}, \mathrm{IBS}^{27}, \mathrm{FOV}^{28}, \mathrm{px}^{29}, \mathrm{HMM}^{30}, \mathrm{FAMS}^{31}$

${ }^{1}$ Single-molecule Förster Resonance Energy Transfer

${ }^{2}$ Alternating laser excitation

${ }^{3}$ Acceptor

${ }^{4}$ Donor

${ }^{5}$ Pulsed-interleaved excitation

${ }^{6}$ Non-coding ribonucleic acid

${ }^{7}$ Deoxyribonucleic acid

${ }^{8}$ Circular dichroism

${ }^{9}$ Isothermal titration calorimetry

${ }^{10}$ Total internal reflection fluorescence (microscopy)

${ }^{11}$ G-quadruplex

${ }^{12}$ Kissing complex

${ }^{13}$ Extended duplex

${ }^{14}$ Accessible volume

${ }^{15}$ (Four-)way junction

${ }^{16}$ Wild type

${ }^{17}$ (FRET state) transition density plot

${ }^{18}$ Time-correlated single photon counting

${ }^{19}$ Aminopurine

${ }^{20}$ Nucleotide

${ }^{21}$ Loop

${ }^{22}$ Electron multiplying charged-coupled device

${ }^{23}$ Full width at half maximum

${ }^{24}$ Bayesian information criterion

${ }^{25}$ Truncated group IIB intron construct for single-molecule FRET, D135: domains 1, 3, and 5 including loops for fluorescent labeling in domains 1 and 4 (L14).

${ }^{26}$ Exon binding site

${ }^{27}$ Intron binding site

${ }^{28}$ Field of view

${ }^{29}$ Pixel

${ }^{30}$ Hidden Marcov modell(ing)

${ }^{31}$ Fluorescent-aided molecular sorting 


\begin{abstract}
More than two decades investigating nucleic acids and ribonucleic acids (RNA) by single molecule Förster Resonance Energy Transfer (smFRET) have passed. It turned out that sample heterogeneity in structure and function of RNA molecules as well as folding intermediates, kinetic subpopulations, and interconversion rates of conformational states of RNA biomolecules, all of which are usually hidden in ensemble type experiments, are often observed characteristics. Besides proteins, metal ions play a crucial role in RNA folding and dynamics, as well as RNA/RNA or RNA/DNA interactions. RNA molecules form discrete conformational intermediates before reaching the native three-dimensional fold, whereby metal ions guide the folding pathway by changing the energetic barriers between local and global minima in the energy landscape. Here we review recent advances in the characterization of the role of metal ions in folding and function of nucleic acid structures by means of smFRET. Subsequently, the workflow of smFRET data analysis is described and exemplified by the metal ion-depending folding and dynamics of the group IIB intron from $S$. cerevisiae and RNA-RNA binding kinetics of this ribozyme's 5'-splice site formation.
\end{abstract}

Keywords: smFRET, RNA folding, RNA metal ion interaction, ensemble heterogeneity, movie processing, smFRET data analysis 


\section{Table of Contents:}

Abstract

1. Introduction

2. Cation-induced changes in ribonucleic acid structure revealed by smFRET

2.1 Secondary structure formation

2.1.1 Three-way junctions

2.1.2 Kissing hairpins

2.1.3 GAAA tetraloop-receptor

2.1.4 Four-way junctions

2.2 Ribozymes

2.2.1 The hairpin ribozyme

2.2.2 Other small ribozymes

2.2.3 RNAse P, group I and group II introns

2.3 Riboswitches and aptamers

2.4 smFRET studies on DNA vs. RNA

2.5 Quadruplexes (GQ)

3. Single molecule movie, image and signal processing

3.1 A guide for smFRET analysis

3.2 Background correction

3.3. sm spot detection

3.4 Co-localization and time trace generation in multi-channel experiments

3.5 sm exclusion rules

3.6 Reducing noise in sm time traces 
3.7 Alternating laser excitation (ALEX)

3.8 Static and dynamic single-molecules

3.9 Modelling the correct number of states

3.10 sm kinetics

3.11 Bootstrapping to access sample variability

4 Conclusions

5 Acknowledgements

6 References 


\section{Introduction}

Since the first reports on single-molecule ( $\mathrm{sm}$ ) microscopy and spectroscopy in the early nineties [1,2], fluorescence-based techniques have undergone a tremendous development in terms of detection and analyzing capabilities. In particular, Förster resonance energy transfer (FRET) as a spectroscopic ruler is nowadays a standard tool for the characterization of biomolecular structure and dynamics [3-5], both on the ensemble and the single-molecule level (smFRET) [6-9]. This technique is based on the distance-dependent energy transfer between two fluorophores attached to the biomolecule of interest, which show a spectral overlap between the emission spectrum of the donor (D) and the absorption spectrum of the acceptor (A) [10]. Usually the FRET efficiency, i.e. the inter-fluorophore distance, is followed over time and/or with changing environmental conditions upon excitation of the donor fluorophore. The application of smFRET to numerous biomolecules including proteins [8,9,11-13], DNA [14,15], and RNA [15,16] yielded valuable insights on intra- and inter-molecular processes on timescales ranging from nanoseconds to hours. Alternating laser excitation (ALEX) and/or pulsed interleaved excitation (PIE) [17,18] were introduced in order to characterize the labeling of the single molecules in terms of fluorophore stoichiometry. Moreover, multi-color FRET experiments are possible and performed to follow multiple inter-domain motions in real time [19-21].

The main advantage of single-molecule methods is their ability to unveil sample heterogeneity, which is usually hidden in ensemble-averaged experiments. Heterogeneity is an inherent property of nature caused by the structural divergence of individual conformations within the same and/or different species of the (bio)molecule. smFRET experiments are versatile and allow to observe also minor occurring species, transient intermediate states [22], and parallel folding pathways including static and dynamic heterogeneity $[23,24]$. The access to (sub)structural information monitored by smFRET is further broadened by the photophysical properties, i.e. changes in the spectrum and in the quantum yield, of the introduced fluorophores as well as noise made by the stochastic nature of photon emission (shotnoise), background noise, read-out noise etc. [24-27], or technical aberrations such as fluctuations in laser intensity [28,29]. Emission spectra, fluorescence lifetime, and fluorescence anisotropy measurements provide information about the local environment of the (bio)molecule, such as the viscosity [30], the dielectric constant [31,32], and 
the local density $[33,34]$. These information are in particular important to explain some variations, e.g. of the conformational dynamics observed in vitro vs. in vivo [35,36].

Ribonucleic acid (RNA) is a polymeric and polyanionic macromolecule essential to all known forms of life, in which it performs uncountable crucial biological roles [37-39]. While genetic information transfer is the best known property [40], RNA is also capable of catalytic activity [41]. Catalytic RNAs are known as ribozymes [42] and were first reported in 1982 by Cech et al. with the self-splicing of the IVS sequence in Tetrahymena thermophila rRNA [43], as well the RNAseP cleavage reaction investigated by Altman and Pace [44]. Today it is estimated that only $3 \%$ of the transcribed RNA is translated into proteins, whereas the remaining $97 \%$ are non-coding RNA (ncRNA) sequences, many of which relate to gene regulation or disease development, giving a whole new dimension and relevance to the RNA presence and function in the cell [45-48].

\section{<insert Figure 1 here $>$}

The primary structure of nucleic acids (NA) results from the order of the monomer-nucleotides in the polymeric chain. The main driving force for RNA folding into secondary structures is base stacking, which increases stability of the sequence: the surface of the aromatic bases is hydrophobic and their association reduces the area exposed to the polar solvent (usually, water) [49]. However, RNA is a negatively charged polyelectrolyte, i.e. a polyanion, with one negative charge per monomer. Its folding therefore requires the presence of positive counter-ions to compensate the repulsion between the phosphate groups of the backbone when bringing them close together in space [52-54]. Indeed, monovalent metal ions such as $\mathrm{K}^{+}$promote the first step of RNA folding to secondary structures, with further compaction and tertiary contacts (i.e., interaction between secondary structures) being built in the presence of divalent cations, such as $\mathrm{Mg}^{2+}$ (Figure 1) [55-57]. The tertiary structure is relevant for biological function (e.g. catalysis, ligand interaction, gene regulation) [58,59] and can be accessed by computational modeling or determined by either X-ray crystallography or NMR spectroscopy as high-resolution bulk methods $[53,60,61]$. Both techniques provide atomistic insights of the three-dimensional structure thus allowing the direct localization of cations bound to nucleobases. NMR requires the metal ion to have a nuclear spin. Numerous physiologically important metal ions can therefore not be directly detected, including $\mathrm{Mg}^{2+}$, which is usually substituted by $\mathrm{Mn}^{2+}$ or $\mathrm{Cd}^{2+}$ [61-63]. X-ray crystallography on the other hand suffers from the higher flexibility of the RNA strand, which renders crystal formation in particular of long single strands and floppy domains impossible. Other 
techniques that are routinely used to characterize RNA folding such as UV thermal melting, isothermal titration calorimetry (ITC), and circular dichroism (CD) do not report on the threedimensional structure itself, but rather on specific features such as thermal stability or the prevalence of specific secondary structure motifs [64-71]. Single molecule techniques and especially smFRET as a low resolution technique combine the determination of structural and functional information at the same time.

The first part of this review is dedicated to smFRET investigations of NA structure formation and its heterogeneities influenced by metal ions. FRET reports on the metal-ion-dependent inter-dye distance requiring the preceding labeling of NAs. Hence, control experiments have been developed and must be carefully chosen to ensure that (i) the metal ions do not influence the photo-physics of the fluorophores and (ii) that the attachment of dyes does not alter the NAs structure and functionality, both leading to misinterpretations [5]. A comparison of different fluorescent labels discussing stability issues etc. would go beyond the scope of this review and hence is given elsewhere [72].

The second part of this review covers the workflow and pitfalls encountered in single-molecule FRET data analysis, in particular for camera-based data acquisition in total internal reflection (TIR) and wide field fluorescence microscopy. The multitude of metal ions (occurring in vivo and being previously studied by NMR and X-ray) associated with RNA folding and function together with the necessity for titration experiments, and to obtain several hundreds of single molecules at each condition to allow statistical analysis yield large data sets. For the subsequent data analysis of these large data sets various software solutions have been proposed over the past years [73]. For camera-based fluorescence detection the software typically comprises spot detection, single molecule tracking, background correction, coordinate transformation and/or co-localization, fluorescence intensity time trace generation, and FRET state discretization. Furthermore, statistical analysis with significance tests and clustering of determined states and state transitions are considered. Choosing a subsample of molecules, fulfilling individually determined criteria of the experimentalist, needs impartial algorithms to avoid biased selections. Herein, the quantification of metal ion-dependent changes in inter- and intra-molecular RNA interactions as for the 5'-splice site formation [74] as well as the folding pathway analysis of the group IIB intron of $S$. cerevisiae $[75,76]$, will be discussed in detail and used to exemplify the analyzing procedures. 


\section{Cation-induced changes in ribonucleic acid structure}

\section{revealed by smFRET}

Two biologically relevant classes of NA exist, deoxy-ribonucleic acids (DNAs) and ribonucleic acids (RNAs). The presence of a 2'-hydroxyl group in the sugar unit of the RNA results in added steric hindrance and further hydrogen bonds with surrounding water molecules or interacting ligands / proteins. Moreover, the 2'-OH gives RNA its inherent instability, as it facilitates selfhydrolysis of the RNA chain by a nucleophilic attack on the adjacent 3'-phosphate. This is the "price to pay" for the higher versatility of RNA.

\section{<insert here Table 1 $>$}

$\mathrm{K}^{+}$and $\mathrm{Mg}^{2+}$ are the most abundant mono- and divalent metal ions in the cytoplasm of human cells [77] and also the most relevant for NA folding (even if $\mathrm{Na}^{+}$and $\mathrm{Li}^{+}$can also fold some RNAs [78]). Both have closed-shell electron configurations and are not redox active. The interaction depends on the ionic radius and charge, which define the charge density, dehydration energy, and coordination sphere. The higher the charge density, the stronger the interaction with water, and the more difficult it will be to dehydrate the metal ion [52]. Being rather small $(\sim 0.65 \AA) \mathrm{Mg}^{2+}$ has a high charge density and is therefore surrounded by six ordered water molecules in octahedral coordination [52,79]. In contrast, $\mathrm{K}^{+}(1.3 \AA$ radius $)$ is accompanied by eight or nine less ordered waters [52]. Divalent cations have thus an intrinsic higher affinity towards NA compared to $\mathrm{M}^{+}$, although the later are present in much higher concentration [80]. A small percentage of metal ions is tightly and specifically bound, whereas the vast majority are more loosely bound and are not located in discrete binding pockets $[62,81,82]$. Important aspects are that (i) cations, in particular monovalent metal ions, are necessary for the formation of RNA secondary structure elements $[60,83]$, albeit divalent metal ions are essential to stabilize short base-paired regions [50] as they occur in RNA tertiary contact interactions exemplified by the splice site formation of d3'EBS1*/IBS1* as an RNA duplex [74], (ii) the exchange rate of divalent metal ions, in particular $\mathrm{Mg}^{2+}$, seems to be much slower in a tight binding pocket than the pure ligand exchange rates suggest [74], and (iii) metal ions can exert their influence on structure and function of an RNA not only through direct coordination, but as well from a distance [84]. Capable to provide an atomistic view, computational studies could show that $\mathrm{Mg}^{2+}$ coordination provides at the same time structural stability and catalytic activity speeding up the reaction rate [85]. Aside from $\mathrm{Mg}^{2+}$ 
recently also further divalent metal ions have been investigated more closely, showing that some

of them even accelerate catalysis of ribozymes compared to $\mathrm{Mg}^{2+}[62,81,86]$ but also disturb the folding [87]. Important thereby seems the different intrinsic affinities towards phosphate groups (Figure 1).

The effect of different metal ions on RNA and DNA was investigated by means of smFRET in fluorescence wide field and TIR microscopy. An overview is given in Table 1 with special attention on the type and concentration range of metal ions. Throughout all investigated RNAs with model character (Figure 2), heterogeneities were observed, quantified and often related to the presence of metal ions, as will be discussed below.

\section{<insert here Figure 2>}

\subsection{Secondary structure formation}

DNA and RNA nucleobases pair predominantly by hydrogen bonding in the canonical WatsonCrick way, which links $\mathrm{C}$ and $\mathrm{G}$ by three and $\mathrm{A}$ and $\mathrm{U}$ by two hydrogen bonds. This results in right-handed double helices as secondary structure, e.g. an A-form RNA (Figure 3A). with slightly different structural parameters to the classical B-form DNA duplex (Figure 3B). The ARNA is wider but more compact with the nucleobases tilted with respect to the helix axis and the sugar conformation in $\mathrm{C} 3$ '-endo, which contrasts to B-DNA being $\mathrm{C} 2$ '-endo. A rare but possible secondary structure of RNA is the left-handed double helix called Z-form (Figure 3C), which only exists under non-physiological high-salt conditions. Guanosine or cytosine rich RNA sequences build four-stranded structures like G-quadruplexes and i-motifs (Figure 3D and 3E). G-quadruplexes are stable under physiological conditions and are mostly found in regulatory regions of DNA [89] or RNA [90] hinting at a possible role in regulating cellular processes as is further discussed in Section 2.4. However, RNA is more often "single-stranded" and folds rather intramolecular upon itself instead of associating to a second strand. As a consequence, numerous secondary structure motifs are formed like hairpins, internal loops, bulges and junctions (Figure 3F-i-vii) and tertiary contacts like loop-loop interactions and pseudoknots (Figure 3F-viii-ix) to achieve functionality. These motifs are part of a complex higher architecture of larger elements including the catalytic core of ribozymes. Elucidating their structural dynamics under relevant conditions is thus crucial for the understanding of the overall RNA activity. In Sections 2.1.1 to 2.1.4 we will discuss representative smFRET investigations of such functional systems in dependence of $\mathrm{M}^{2+}$ ions. 


\section{<insert here Figure 3>}

\subsubsection{Three-way junctions}

The RNA triple-helix junction, commonly known as three-way junction (Figure 3F-vii), is, e.g. found in transfer messenger RNAs (tmRNAs) [96], in the hepatitis C virus internal ribosome entry site (IRES) [97], in the catalytic domain of the Bacillus subtilis RNAse P [98], and in a modified structure of the 16s RNA junction studied by Kim et al. [99], among others. The first three-way junction investigated by smFRET was the RNA scaffold of the Escherichia coli 30S ribosomal subunit [100]. The formation of the ribosomal subunit consists of twenty-one successive proteinRNA binding events, each of them inducing RNA folding to form the next protein binding site (PBS). Two helices of the RNA were labeled with the donor/acceptor pair Cy3/Cy5 and fluctuations in FRET were reported to be conformational changes upon binding of the very first ribosomal protein $\mathrm{S} 15$ or upon increase of $\mathrm{Mg}^{2+}$ concentration. RNA-protein binding affinities were determined from the averaged data from single molecule experiments and were in accordance with bulk gel electrophoretic assays performed with unlabeled RNA. This observation exemplifies a fundamental principle of sm studies, which yield the ensemble average upon averaging of the single molecule information for both the observation time and the number of detected molecules (ergodicity) [101].

The introduced labeling scheme allowed one to visualize two distinct conformations of the construct: The two helices are either far from each other in the so-called original state or they are collinear in the fully-folded state. The structure of the RNA three-way junction is strongly dependent on $\mathrm{Mg}^{2+}$ with a higher concentration increasing the proximity of the two helices, which already starts at relatively low $\mathrm{Mg}^{2+}$ concentration of $100 \mu \mathrm{M}$. High $\mathrm{Mg}^{2+}$ concentrations induce the same folded structure as the protein S15, but the binding equilibrium is reached much faster if the folding is initiated by the addition of cations. Furthermore, $\mathrm{Mg}^{2+}$ yields a progressive shift of the FRET values from the unfolded to the native FRET state, contrasting with the population change of two FRET states by changing the protein concentration. This observation revealed a structural or kinetic heterogeneity in the presence of $\mathrm{Mg}^{2+}$, thus refuting a simple two-state mechanism. In accordance to this, the authors predicted a cation charge-screening mechanism rather than a site-specific binding of the cations. In the latter case, a wide distribution of binding affinities is assumed, considering that the binding/unbinding events are faster than the temporal resolution. Although the origin of the observed heterogeneity was not unambiguously determined, 
it exemplifies that this is a striking feature of single RNA molecule studies, which yield folded intermediate subpopulations $[22,96,98,102,103]$ or heterogeneities of the conformational space $[21,75,104,105]$ and/or the reaction kinetics [106-108].

By means of slow $(200 \mathrm{~ms})$ or fast $(50 \mathrm{~ms})$ buffer exchange experiments (addition of $1 \mathrm{mM}$ $\mathrm{Mg}^{2+}$-containing or $\mathrm{Mg}^{2+}$-free buffer), the authors could further demonstrate how single-molecule folding in RNA occurs reversibly on a sub-second timescale. While $\mathrm{M}^{2+}$ facilitate the transition to the active state $[75,109]$, small modifications of the local structure close to the junction or loop structures accelerate folding by several orders of magnitude. In that way, the active state can be reached already at sub-millimolar $\mathrm{Mg}^{2+}$ concentrations, i.e. physiological conditions, providing valuable information for the understanding of in vivo mechanisms $[109,110]$.

\subsubsection{Kissing hairpins}

This motif results from the dimerization of two canonical hairpin loops and is found for example in group II introns ( $\alpha-\alpha$ ' interaction) [111], tRNAs [112], the RNA dimerization initiation sites (DIS) within the dimer linkage structure (DLS) of the two copies of HIV-1 genomic RNA [113], and riboswitches (aptamer formation) [114-117]. The resulting so-called kissing complex (KC, Figure 3F-viii), is very stable and can constitute a kinetic trap during folding. A kissing complex can even prevent reaching the native fold, i.e. the operative and functional form of the RNA [118]. Previous single-molecule studies aimed at characterizing the thermodynamics of the $\mathrm{KC}$ formation of two RNA hairpins (HP1 and 2) and its transition to an extended duplex (ED) of both RNA strands in order to reveal the cofactor-assisted refolding mechanism occurring in vivo, as well as the kinetic trap formation. Like protein-stability studies in living cells [119], the single KC formation and the transition to ED has been investigated using short infrared (IR) laser pulses that heat the sample locally to temperatures higher than $50^{\circ} \mathrm{C}$ [103]. Such high temperatures melted the short sequences and induced subsequent refolding into the ED. One of the hairpins was labeled with $\mathrm{Cy} 3$ at the 3 '-end and with a biotin moiety at the 5'-end for surface immobilization. The second hairpin was labeled with Cy5 at the 5'-end and allowed to diffuse freely in solution. Three FRET values were observed: A zero FRET state corresponding to the undocked hairpins, an intermediate FRET state assigned to the kissing hairpin complex, and a high FRET state observed upon formation of an extended duplex. The KC to duplex conversion [120,121] was thus confirmed on the single molecule level. The relative occurrence of the particular three FRET states at different temperatures allowed one to determine the activation energy barriers for the 
dissociation and the refolding to be $27 \mathrm{kcal} \mathrm{mol}^{-1}$ and $38 \mathrm{kcal} \mathrm{mol}^{-1}$, respectively. The refolding activation energy was higher, proving the ED formation without temperature jumps to be rare. This explained why the folding of biomolecules possessing two hairpins can be kinetically trapped, favoring the formation of a $\mathrm{KC}$ [103]. In a follow-up study [118], stabilization of the KC by $0.6 \mathrm{kcal} \mathrm{mol}^{-1}$ and a threefold decrease of the dissociation rate was achieved upon replacement of U-A by G-C in the loop; destabilization by $2 \mathrm{kcal} \mathrm{mol}^{-1}$ was observed upon the introduction of a single base pair mismatch. The kinetic analysis of the $\mathrm{KC}$ formation/dissociation showed that the stabilization and/or destabilization affected only the dissociation kinetics while the association rates of all hairpin variants were similar. In addition, the disruption of a central base pair or reducing the loop size from 7 to 5 base-pairing nucleotides inhibits the transient formation of the KC. Again, the transition from KC to ED is rare and the authors argue that in biological systems protein cofactors facilitate the transition from the kissing complex to the extended duplex by using the protein-RNA-binding energy to break the coaxial stacking of the $\mathrm{KC}$. This was shown in the presence of divalent cations, where the DIS kissing dimer is kinetically trapped and converts to the extended duplex isoform only upon addition of the nucleocapsid protein (NCp7) [122]. A recent smFRET study of HIV-1 DIS forming a KC and/or ED revealed a bent intermediate state that requires $\mathrm{Mg}^{2+}$ [123]. Surprisingly, this intermediate state shows the same high FRET value as the extended duplex $[103,118]$ and is in equilibrium with the coaxially stacked conformation until the extended duplex is eventually irreversibly formed showing a constant high FRET state [123]. This exemplifies that understanding FRET state and or kinetic heterogeneity is essential to understand the folding process of RNAs. Metal ion titrations revealed a long-lived (stable) KC only formed in presence of higher $\mathrm{Mg}^{2+}$ concentrations $(>0.5 \mathrm{mM})$. High amounts of monovalent metal ions $\left(\left[\mathrm{K}^{+}\right]>300 \mathrm{mM}\right)$ compete with the binding of divalent metal ions as they recover the population of the short-lived KC. However, the necessary high amount of $\mathrm{K}^{+}$indirectly proves the binding site for $\mathrm{Mg}^{2+}$ to be tight and specific. The authors close with promising sm studies to further elucidate the role of NCP7 in the dimerization pathway, thus, not leaving RNA molecules as "pure" substrate to understand the RNA-protein interaction but understanding the RNA structure and function in the presence of proteins.

\subsubsection{GAAA tetraloop-receptor}

The docking of the GAAA tetraloop with a specific receptor sequence forms a tertiary motif that is widely abundant in RNAs [124], such as the P4-P6 domain of Tetrahymena group I introns [125]. 
Nesbitt et al. reported a detailed analysis of the tetraloop-receptor interactions of the hairpin ribozyme by means of time-resolved smFRET studies $[104,126]$. The GAAA tetraloop was linked to the receptor domain by a seven-nucleotide spacer $\left(\mathrm{A}_{7}\right)$ in order to reduce the accessible volume (AV) while maintaining the freedom of motion for this region. The exchange of the $A_{7}$ linker by a $\mathrm{U}_{7}$ linker demonstrated that the linker sequence does not significantly alter the docking dynamics. Tetraloop-receptor docking events were observed at physiological monovalent ion concentration in the absence of $\mathrm{Mg}^{2+}$ (dissociation constant for the docking equilibrium $K_{\text {dock }}=0.49 \mathrm{mM}$ ). The docking and undocking rate constants calculated from the single molecule data revealed heterogeneity in the folding kinetics: Two thirds of the identified molecules showed relatively rapid docking/undocking transitions, while dynamics were rare in the remaining population. $\mathrm{Mg}^{2+}$ dependent docking/undocking rates were fitted by a Hill-type equation [99], yielding an apparent dissociation equilibrium constant $K_{\mathrm{Mg}(\mathrm{II})}$ of $1.84 \mathrm{mM}$ and a Hill coefficient of $\sim 1$, leaving tetraloop-receptor docking independent of the $\mathrm{Mg}^{2+}$ binding. The tetraloop docking rates determined for the P1 helix in Tetrahymena group I intron were smaller than for GAAA tetraloop comprising the $\mathrm{A}_{7}$-linked emphasizing the importance of close proximity for RNA-RNA interactions.

\subsubsection{Four-way junctions}

The hairpin ribozyme consists of two major structural elements: a four-way (4W) junction and two internal loops from adjacent helices [127]. Tan et al. studied the loop-free four-way junction, the so-called minimal hairpin ribozyme, using smFRET [109]. Based on previous bulk studies of the ribozyme, which proposed an apparent two-state folding pathway [128], they quantified the $\mathrm{Mg}^{2+}$-dependent folding kinetics of the construct. Strikingly, the sm data with a time resolution of $50 \mathrm{~ms}$ revealed the presence of an intermediate state, the so-called proximal state. The quantification of the transition rates between the distal (or unfolded) and the proximal state were determined by cross-correlation of the donor and the acceptor fluorescence intensity signals [99]. The transition rates increased by three orders of magnitude upon reducing the $\mathrm{Mg}^{2+}$ concentration from $50 \mathrm{mM}$ to $0.1 \mathrm{mM}$. The observed acceleration was assigned to an extensive facility of the ribozyme to form its active site at physiological $\mathrm{Mg}^{2+}$ concentration. In accordance, conformational changes of the hairpin ribozyme were followed by smFRET during substratecleavage assays. They observed (i) the folding of the ribozyme to be spontaneous upon addition of $\mathrm{Mg}^{2+}$ and (ii) the molecule remains folded after the cleavage reaction $(\sim 150 \mathrm{~s})$. After this delay, 
the folding rates were calculated from the dwell-time distributions of 83 dynamic molecules of reoccurring dynamics. The kinetic analysis yield a pronounced heterogeneity for the ribozyme rather than for the $4 \mathrm{~W}$-junction. This discrepancy was attributed to the additional loop-loop interaction that can only occur in the ribozyme but not in the loop-free derivative. Accordingly, the average dwell-times of the distal and proximal states of the $4 \mathrm{~W}$-junction exhibited a narrower distribution than the unfolded and native states of the hairpin ribozyme. The authors argue that the fast interconversion rates between two unfolded conformations at low $\mathrm{Mg}^{2+}$ concentration is exploited by the hairpin ribozyme to reach its folded conformation via a discrete but transient intermediate state. The latter was accessible by decreasing the binning time and performing a separate investigation of the $4 \mathrm{~W}$-junction at $\mathrm{Mg}^{2+}$ concentrations below $1 \mathrm{mM}$. However, heterogeneity in the determined kinetic rates was reported by the authors in the presence of $\mathrm{Mg}^{2+}$, as well as in control experiments with $\mathrm{Na}^{+}$and with PEG instead of BSA as surface passivation.

\subsection{Ribozymes}

RNAs with catalytic activity are called ribozymes. Their specific interaction with metal ions plays a crucial role in their folding and catalytic activity $[86,129,130]$ as well as in their conformational heterogeneity $[74,98,131]$ as is exemplified in the below sections.

\subsubsection{The hairpin ribozyme}

The hairpin ribozyme originates from the autocatalytic negative strand of the tobacco ringspot virus satellite RNA [132] and has been investigated by several independent smFRET studies $[102,107,109,133]$. Two minimal sequences of the satellite RNA comprise a 50-nt catalytic RNA sequence of the two independently folding helix-loop-helix domains A and B and a 14-nt substrate RNA sequence, respectively [107,134]. Zhuang et al. demonstrated that the labeling of domains A and $\mathrm{B}$ with the FRET pair Cy3 and Cy5 allows to follow the putative reaction pathway of single immobilized hairpin ribozyme molecules via smFRET [107]. Three distinct FRET states were observed and ascribed to be the undocked, docked, and substrate-released state, respectively.

In a more detailed study, the reaction pathway of the hairpin ribozyme was shown to have five reversible steps: Substrate binding, hairpin docking, cleavage reaction, hairpin undocking, and final release of the cleaved substrate, the 3'- and 5'-products [128]. The forward and backward transition rates of the four intra- and intermolecular state changes were determined by means of smFRET. When the substrate is bound to domain A, the hairpin ribozyme adopts a stretched 
(undocked) conformation. The FRET efficiency increases upon docking of domain A and B (docked). In the docked state the cleavage reaction might occur, but is spectroscopically hidden as the apparent FRET value does not change. Therefore, the rates for substrate cleavage and ligation have to be determined indirectly. The fraction of cleaved molecules with respect to the observation time was obtained from bulk cleavage assays, subject to the forward and backward reaction paths described above. The rates of the underlying reactions are determined by smFRET and by fluorescence based kinetic analysis [107]. Knowing these rates and solving a system of rate equations enabled to derive the cleavage and ligation rate from bulk experiments, yielding a ligation rate about twice as fast as the cleavage rate. The docking and undocking rates of the cleaved loop were determined by offering saturating concentrations of the already cleaved substrate fractions, the 3'- and 5'-product, yielding similar rates like in the case of binding the uncleaved substrate. The docking process proves to be homogeneous, whereas the undocking is heterogeneous. The respective undocking rates span 4 orders of magnitude (from $1 \mathrm{~ms}^{-1}$ to $1 \mathrm{~s}^{-1}$ ). The rate for the 5'-product release was determined by cross-correlation of donor and acceptor fluorescence intensity trajectories since both the 5'-product bound and the released state exhibited only slightly different FRET values making the state determination erroneous. The dissociation equilibrium and rate constants of the 3'-product were determined by attaching the fluorescence quencher dabcyl to the 3'-end of the 3'-product itself (3'P-Dabcyl) and subsequently applying dwell-time analysis on the fluorescence intensity intermittence of the acceptor fluorophore Cy5. The association and dissociation rate of both cleavage products, the $3^{\prime}$ and 5', differ only slightly for the dissociation rate, but by a factor of 5 for the association rate. In addition, the authors report on a strong memory effect, i.e. hairpin ribozyme molecules still behave similar after a few hours of waiting time with respect to their undocking rates. Thus, the heterogeneous cleavage kinetics in catalytic RNAs may result from such (kinetic/structural) subpopulations and their distinct rates.

This assumption appeared to be a general phenomenon for many catalytic RNAs [75,135]. As discussed for various RNA model systems (vide supra), Tan et al. demonstrate that millisecond fluctuations between so-called distal and proximal conformations in the four-way junction of the hairpin ribozyme decelerate upon increase of the $\mathrm{Mg}^{2+}$ concentration from the sub-millimolar to the millimolar range [109]. Faster dynamics in the sub-millimolar range seem to operate as a folding enhancer, ensuring efficient folding into the catalytically active conformation of the hairpin ribozyme. Bokinsky et al. later discussed the role of $\mathrm{Mg}^{2+}$ and $\mathrm{Na}^{+}$ions on the docking and undocking rates between the undocked transition states [133]. From theoretical modeling and the 
transition rate dependency of $\mathrm{Mg}^{2+}, \mathrm{Na}^{+}$, and a combination of both, they propose two transition states. One is represented by the contact model, where domains A and B are in contact, and another by a model where the two domains are only slightly separated. In the presence of both, $\mathrm{Na}^{+}$and $\mathrm{Mg}^{2+}$, the saturation effect of $\mathrm{Mg}^{2+}$ on the undocking rate is present at a significantly higher concentration than in the absence of $\mathrm{Na}^{+}$, indicating a competitive binding of both metal ions to unspecific metal ion binding sites on the RNA. Moreover, they discuss the role of the water solvent on the stabilization of the docked state with respect to the undocked state. This effect is supposed to arise from an increased polarization of water molecules close to more compact RNA molecules due to their stronger electrostatic field. Studies mutating the RNA sequence of the ribozyme showed that although the inter-domain base pairing is not yet formed in the transition state, functional groups of different domains begin already to form tertiary contacts, accelerating the folding process.

Rueda et al. presented smFRET studies combined with ensemble cleavage assays and kinetic simulations to demonstrate the influence of modifications of essential groups, which are remote from the catalytic site/core, on the rate constants [102]. Cleavage rates were determined from single-turnover reactions and the time course was subsequently modeled by solving a system of rate equations and taking a subspecies of different undocking rates into account. Like in [107] the docking and undocking rates were determined by dwell-time analysis taking the heterogeneity of the undocking process into account. The five ribozymes modified at specific functional groups still exhibit a similar fractioning into non-interchanging subpopulations of molecules with different undocking rate constants. Thereby, those of the modified ribozymes showing accelerated docking rates also show multiple docking rates and are thus exhibiting heterogeneity. Remarkably, the modified ribozymes with modifications distant from the catalytic site display not only altered docking and undocking rates but also different catalytic activity. Rueda et al. suggest that a network of coupled molecular motions connects distant parts of the RNA with the catalytic site, in a fashion akin to some previously described enzymes [136].

\subsubsection{Other small ribozymes}

De Silva et al. used smFRET to study the formation of a prototype drug-sensing aptazyme derived from the theophylline aptamer and the hammerhead ribozyme. The catalytically active state with distal stems I and III being close is accessed in the theophylline bound and less frequently in the ligand free state [137]. They also showed that the cleavage activity depends on the $\mathrm{Mg}^{2+}$ 
concentration. Rapid local theophylline binding to the aptamer domain upon site-specific 2aminopurine (AP) labeling was demonstrated but leads to low allosteric signal transduction into the ribozyme core.

Compared to the wild type (wt), the so-called minimal hammerhead ribozyme motif cleaves only in 10-fold higher $\mathrm{Mg}^{2+}$ concentrations compared to physiological conditions. Mc Dowell et al. investigated the wt and two variants of the hammerhead ribozyme with partially or fully disrupted Stem I-II loop-loop-interaction sequences by a combination of smFRET and molecular dynamics (MD) simulations [138]. The folding paths trajectories were discretized by Hidden Markov based dwell-time analysis [139] and subsequently plotted as transition density plots (TDP). Consequently, differences in the folding pathway and the transition kinetics of each hammerhead ribozyme variant were directly comparable. Their TDPs served as fingerprints and revealed detailed structural dynamics hidden in the broad FRET distributions. The loop-loop interaction sequences and the $\mathrm{Mg}^{2+}$ concentration dependency were suggested to bias the free energy landscape of the folding process towards more disparate stem-stem distances. This allows the hammerhead ribozyme to access new conformations and to populate rare conformations, respectively [138].

\subsubsection{RNAse P, group I and group II introns}

Bulk and single molecule studies of metal ion dependent folding and function-studies of small RNAs together with the introduction of site specific mutations are well established. In the past few years, such studies have also been transferred to study larger RNAs on the single molecule level, as these strictly require divalent metal ions for folding and function [140]. In particular the influence of the specific and unspecific binding of domains and metal ions, respectively, gained attention to disentangle the folding pathway that the RNA follows to reach the native, i.e. active state.

Among the first smFRET studies on large RNAs is the report on the Tetrahymena ribozyme, a $400 \mathrm{nt}$ long RNA derived from a self-splicing group I intron [141]. This ribozyme catalyzes the cleavage of its substrate. The substrate is 3'-end labelled with Cy3, while Cy5 is attached to the 3'end of the tether allowing to follow the ribozyme-substrate docking kinetics. Docking is hampered upon modifying the 2'-hydroxyl group of residue U-3 on the substrate, which forms a tertiary contact to the ribozyme. Adding $\mathrm{Mg}^{2+}$-lacking buffer to the sample induces immediate substrate undocking. Indeed, a $5 \mathrm{mM} \mathrm{Mg}^{2+}$ concentration is required to establish efficient docking. The 
guanosine-dependent reaction kinetics of the smFRET experiments are in good agreement with ensemble measurements. Within the overall folding process, intermediate folding states and in particular multiple folding pathways were observed: A first fast folding pathway from the unfolded to the native state through an intermediate, with a rate constant of $1 \mathrm{~s}^{-1}$; a second pathway going through a different intermediate and having a slower rate $\sim 0.16 \mathrm{~s}^{-1}$; and a third pathway comprising a misfolded state and yielding a very slow rate of $\sim 10^{-5} \mathrm{~s}^{-1}$. This case exemplifies perfectly the potential of single molecule studies to reveal the sample variability or heterogeneity hidden in ensemble measurements.

Sattin et al. reported on tertiary contact cooperativity effects within the independently folding P4P6 domains derived from the Tetrahymena thermophila group I intron [142]. The two helices, P4 and P6, are basically connected by a junction (J5/5a), and further interact by two tertiary contacts, the metal core/metal core receptor (MC/MCR) and the tetraloop/tetraloop receptor (TL/TLR). Both have been separately mutated to investigate the tertiary contacts independently from each other. The wt and the modified RNAs exhibit only direct transitions from the unfolded to the folded conformation as observed by the FRET histogram always showing a bimodal distribution. The TL-mutated variant reaches a lower FRET value than the wt and MC mutated variant. This finding was attributed to the fluorophore label position, which is close to the tetraloop contact sites and is no longer stabilized within the TL-mutated construct. The cooperativity is quantified from the equilibrium constants obtained from the respective FRET histograms. Calculating the ratio of the equilibrium constants of the wt and the respective mutants a decreased stability of 120 -fold and 24-fold for the mutated TL/TLR and the mutated MC/MCR interaction are obtained, respectively. Moreover, $\mathrm{Mg}^{2+}$-dependent folding was observed by smFRET and hydroxyl radical footprinting with comparable half-point $\mathrm{Mg}^{2+}$ concentration values of 2.4 and $2.6 \mathrm{mM}$ for the wildtype, 18 and $13.5 \mathrm{mM}$ for MC-mutant as well as $41 \mathrm{mM}$ in both experiments for the TL mutant. This was in line with the previous stabilization argument of the tetraloop, which has a stronger stabilization effect and thus needs a higher metal ion concentration to compensate the TL/TLR tertiary contact mutation.

Among the largest RNA molecules studied by means of smFRET is the $255 \mathrm{nt}$ long RNase P from Bacillus subtilis [98]. Scherer et al. found two previously undetermined folding intermediates via $\mathrm{Mg}^{2+}$-dependent FRET efficiency histogram analysis, by labeling their RNA with Cy3 at the 3'end and fluorescein at the 5 '-end. The analysis of FRET trajectories at intermediate $\mathrm{Mg}^{2+}$ 
concentrations revealed five classes of molecules based on three different FRET states. Furthermore, the initial $\mathrm{Mg}^{2+}$ concentration turned out to be crucial since distinct folding pathways and faster kinetics are induced upon starting from $\mathrm{Mg}^{2+}$-free or sub-millimolar pre-equilibrium conditions as already observed for the Tetrahymena group I intron [143,144]. Only one year later, a conceptually new labeling strategy for large RNAs using oligonucleotide hybridization was presented on the same RNase P from Bacillus subtilis by Smith et al. [145]. The strategy was to replace a modular hairpin loop by a functionally unimportant hairpin in the RNA sequence and to hybridize it to a fluorophore-containing DNA oligonucleotide. The authors showed that the loop insertion only minimally perturbs the structure and function of RNase P. Using Alexa488- and Cy5-labeled 20-nucleotide-long oligonucleotides allowed $\mathrm{Mg}^{2+}$-dependent investigation of the RNA folding by smFRET. In periodic $\mathrm{Mg}^{2+}$ jump experiments the authors found an energy barrier in the absence of $\mathrm{Mg}^{2+}$ where RNase $\mathrm{P}$ molecules are interconverting between two different conformations [146]. Heterogeneity of transition kinetics might thus be caused by molecules of comparable FRET states and comparable $\Delta G$. The free enthalpy is reduced upon adding $\mathrm{Mg}^{2+}$ $(>0.1 \mathrm{mM})$ [146]. The bi-exponential fitting model describes the cumulative dwell-time histograms best. The observed kinetic heterogeneity is supposed to be due to two distinct conformations, visible as FRET states and undergoing intramolecular interactions. However, further domain interactions of un-labelled domains may affect the dynamics of the observed states too [97]. This predicted RNA folding intermediates are supposed to be well-defined secondary but ill-defined tertiary structures already suggested by small-angle X-ray scattering (SAXS) measurements of the C-domain [147] and of other RNAs [148,149].

The folding and activity of the group IIB intron from $S$. cerevisiae is strictly $\mathrm{M}^{2+}$ dependent $[130,150]$. A minimal well-established construct comprising domains 1, 3, and 5 and labeled with Cy3 and Cy5 via loop insertion and oligonucleotide hybridization was investigated by smFRET [75]. The increase of $\mathrm{Mg}^{2+}$ ions facilitates the formation of the native (catalytically active) state and increases structural dynamics. Four on-pathway conformations are observed in the dynamic equilibrium. At $\mathrm{Mg}^{2+}$ concentrations lower than $1 \mathrm{mM}$ most molecules remain static in a low FRET state called extended intermediate. The number of dynamic molecules interconverting to the more compact conformations increases upon increasing the $\mathrm{Mg}^{2+}$ concentration. The determined dissociation constant $K_{\mathrm{Mg}(\mathrm{II})}=41.4 \mathrm{mM}$ corresponds to the value obtained by bulk folding and cleavage assays. While the forward rate from the extended intermediate to the folded conformation is slightly decreased upon addition of $\mathrm{Mg}^{2+}$, the backward rate decreases 
considerably, yielding an enhanced stabilization of the folded conformation. The most compact conformation, which is ascribed to be the native state, emerges at $>20 \mathrm{mM}$ but is only transiently populated showing a backward to forward folding rate ratio of 24 . The addition of the $17 / 7$ substrate comprising the 5'-splice site of the group IIB intron stabilizes the native state. This effect was even more pronounced than upon addition of higher $\mathrm{Mg}^{2+}$ concentrations. Nonetheless, the population of the native state remains transient in the presence of the substrate. In a later study Karunatilaka et al. found the DEAD-box protein Mss116 in combination with ATP to be a crucial co-factor to considerably stabilize the native state of the ribozyme [151]. The kinetics of the group II intron exhibit heterogeneous subpopulations upon addition of $\mathrm{Mg}^{2+}$. Both, static and dynamic molecules, have been observed without changing the experimental conditions, and the dwell-time distributions show a bi-exponential behavior, thus heterogeneity in the state transition dynamics [75]. In a follow up study, the authors investigated the influence of $\mathrm{Ca}^{2+}$ on the folding pathway of the group II intron [22]. A second subpopulation was emerging when the concentration of $\mathrm{Ca}^{2+}$ increased from 0 to $10 \mathrm{mM}$ in a $\mathrm{Mg}^{2+} / \mathrm{Ca}^{2+}$ mixed solution, keeping the total divalent metal ion concentration constant at $100 \mathrm{mM}$. While the first subpopulation behaves similarly to the one reported before [75], the second was attributed to a misfolded state M (observed as new FRET state). Earlier bulk experiments stated that $\mathrm{Ca}^{2+}$ inhibits the catalytic activity of group II introns [152], but the smFRET study revealed for the first time to which extent the RNA molecules transit into a misfolded, catalytically inactive conformation upon increase of the $\mathrm{Ca}^{2+}$ concentration.

\subsection{Riboswitches and aptamers}

Riboswitches are complexly folded RNA domains located in the 5'-untranslated regions (UTR) of bacterial mRNAs that serve as specific receptors for metabolites and regulate gene expression. They usually act in cis $[153,154]$, but are considered to also act in trans [155], and are additionally found in the 3'-UTR in plants [156]. Riboswitches are composed of two domains, a ligand-binding aptamer that adopts a compact three-dimensional binding conformation to bind the ligand [157] and the expression platform that performs a structural change in order to inhibit the translational or transcriptional machinery. We focus on the heterogeneity investigated by smFRET, exemplifying the switching behavior of riboswitches. An excellent review on riboswitches and their investigation at the single molecule level can be found elsewhere [158].

smFRET measurements in solution under physiological conditions were applied to investigate the specific structural changes to regulate gene expression and to identify RNA subpopulations in 
heterogeneous mixtures. Several smFRET investigations of riboswitches revealed $\mathrm{Mg}^{2+}$-induced partially folded states, which seem to be kinetic intermediates appearing before the final ligandbound native state.

Single-molecule FRET analysis of the $p b u E$ adenine riboswitch revealed that the metal iondependent folding pathway includes a discrete intermediate state, which was not identified in previous ensemble experiments. The increased FRET values in presence of $\mathrm{Mg}^{2+}$ (above $50 \mu \mathrm{M}$ ) are rationalized by the formation of the tertiary L2-L3 loop-loop interaction, which is stabilized upon adenine binding. Therefore, adenine increases the folding rate and significantly reduces the heterogeneity of the folding and unfolding rates between different molecules $[159,160]$.

A second purine riboswitch, the xpt Guanine riboswitch, was investigated by smFRET. Three variants were synthesized to compare the three RNA helices (P1-P3) with dyes positioned on each of the helices. Movements of the helices were observed by either an increase or a decrease in the FRET value depending on whether the helices move farther apart or come closer together. Through monitoring of all three distance relationships, a multistep folding model was derived, which shows that in the unfolded state the P2 helix switches between two conformations and both the distance between P2-P1 and P2-P3 changes. The single-molecule time traces demonstrate rapid switching between the low and high FRET-value. The average dwell-times for the high and the low FRET states of both P1-P2 and P2-P3 are comparable. When either $\mathrm{Mg}^{2+}$ or guanine (G) concentrations are increased the folded state is formed. In this state the P2 and P3 helices are held together by loop-loop interactions, while P1 and P3 must change their relative orientation to adopt the conformation observed in the crystal structure [161]. These structural changes require a higher concentration of $\mathrm{Mg}^{2+}(>1 \mathrm{mM})$ in the absence of $\mathrm{G}$, confirming that $\mathrm{G}$ helps to adopt a folded conformation at physiologically relevant concentrations around $1 \mathrm{mM} \mathrm{Mg}^{2+}$ [162].

The involvement of $\mathrm{Mg}^{2+}$ ions on RNA folding was further demonstrated by smFRET folding studies of the pre-queuosine class II (preQ1-II) riboswitches [163], the S-adenosylmethionine type II (SAM-II) and the larger SAM-I riboswitches. Focusing on SAM-II, addition of $\mathrm{Mg}^{2+}$ stabilizes the high FRET state leading to an equal distribution of an open (low-FRET) and a compacted state (high-FRET). In presence of SAM the high FRET state of the riboswitch dominates. Therefore, $\mathrm{Mg}^{2+}$ seems to induce a compact fold, which is further stabilized by binding of SAM to the previously formed ligand-competent conformation, suggesting the formation of the compact pseudoknot conformation observed in the crystal structure $[164,165]$. 
Unlike purine, preQ1 and SAM riboswitches, the cyclic diguanylate riboswitch (c-di-GMP) shows four distinct dynamic populations for the free RNA. Two populations were either docked or undocked, identified by low or high static FRET ratios. The remaining two conformations fluctuate between docked and undocked but prefer to be in one of this states, identified by either low or high FRET ratios with small fluctuations to the other FRET state. Upon addition of $\mathrm{Mg}^{2+}$ and c-di-GMP, smFRET time trajectories show transitions to a stable docked population for every observed state. Further smFRET mutant analysis reveals that $\mathrm{Mg}^{2+}$ specifically mediates the binding to c-di-GMP, as ligand recognition is accelerated by formation of tertiary interactions distal to the c-di-GMP binding site [166].

smFRET was used with the lysine riboswitch to identify discrete populations involved in the folding transitions induced upon lysine binding. A model FRET system has been developed to accurately measure the dissociation constant and to provide an insight into the rate constants for the opening and closing of the aptamer domain. $\mathrm{Mg}^{2+}$ ions help to decrease the lysine dissociation constant, while at the same time increasing the FRET efficiency and implying that the lysine riboswitch can fold in absence of the ligand. Dwell-time analysis showed that the aptamer remains closed after ligand binding until the expression platform is synthesized. Therefore the regulatory response is proposed to be controlled by the distribution of the bound and unbound populations of lysine and not by the kinetics of closing and opening events [167].

Recently, Holmstrom et al. used smFRET to observe conformation dynamics of a hydroxocobalamin $(\mathrm{HyCbl})$ binding riboswitch (env8HyCbl) [117]. Their studies revealed that the undocking rate associated with the disruption of a long-range $\mathrm{KC}$ interaction is decreased upon binding of the HyCbl ligand. They showed that in the presence of $\mathrm{Mg}^{2+}$, $\mathrm{HyCbl}$ binding preferentially stabilizes the $\mathrm{KC}$, i.e. the docked conformation, and the Shine-Dalgarno sequence enabling ribosome binding is sequestered, thus disabling translation. They found the $\mathrm{KC}$ formation to occur also in the absence of $\mathrm{HyCbl}$ and to depend on the presence of $\mathrm{Mg}^{2+}$. The ligand binding predominately occurs when the $\mathrm{KC}$ is in the undocked state, but $\mathrm{KC}$ docking/undocking also occurs while $\mathrm{HyCbl}$ is bound and is still dependent on $\mathrm{Mg}^{2+}$. Thus, the riboswitch is stabilized in the docked conformation upon ligand binding and the presence of $\mathrm{Mg}^{2+}$ but still able to release the ligand, i.e. by reducing the ligand concentration, which in turn allows to restart translation. This is a striking feature in nature to enable gene regulation on the RNA level without losing substrate nor functional RNA. 
We have seen that in riboswitches, metal ions help to pre-organize a compact conformation, which is further stabilized by ligand binding. Fluorescence resonance energy transfer is commonly used to investigate these conformational changes and to establish folding pathways and dynamics in presence of metal ions and/or of the corresponding ligand. smFRET analysis provided additional insights into structural dynamics of individual molecules to reveal and correlate distinct folding intermediates.

\section{4 smFRET studies on DNA vs. RNA}

Studies of DNA with fluorescence-based single-molecule methods, especially FRET, are well established and have gained exhaustive attention in the past decades [7,168,169]. In particular DNA interactions with proteins have been investigated using FRET [170,171]. Among one of the first reports was the visualization of single a RNA polymerase sliding along DNA [15]. The relaxation, conformational fluctuations, and ionic effects on the elasticity of the DNA substrate were amongst the research topics investigated on single DNA molecules by optical microscopy and spectroscopy in the 1990s [15,172,173]. The possible role of DNA conformational fluctuations in the process of RecA binding to a single double-stranded DNA molecule was subsequently reported $[174,175]$. Thus, DNA often serves as substrate rather than as the molecule of interest in these studies. One of the important investigated technical features was the impact of DNA nature on a preferred dye orientation. The dye orientation is a necessary correction allowing FRET to be used for measuring absolute distances rather than apparent FRET values [176,177].

In many experiments labeled DNA was used as a model to establish new methods based on a wellstudied system and in terms of well-controlled smFRET. Two major representatives are dual-color fluorescence cross-correlation spectroscopy for multicomponent diffusional analysis in solution [178] and alternating-laser excitation (ALEX) of single molecules to facilitate molecule sorting and the analysis of structure and interactions [17]. The extension of smFRET to four-colors to visualize energy-transfer paths on DNA origami has been recently demonstrated [179]. However,

the basic rules governing the process of sequence recognition through base-pairing remain elusive to this day. Cisse et al. used smFRET to precisely quantify the annealing and melting rates of two untethered strands of DNA in a porous vesicle. Analyzing the on- and off-rates depending on the mismatch position in the DNA, the authors determine their respective $K_{\mathrm{D}}$ 's and suggest that seven contiguous base pairs are needed for rapid annealing of DNA and RNA [180]. Additionally the increased stability of the DNA strands upon increase of $\mathrm{Na}^{+}$concentration from 5 to $50 \mathrm{mM}$ was 
directly related to the higher docking rates. Up to three orders of magnitude difference in the dissociation constant $K_{\mathrm{D}}$ were observed depending on the mismatch position [180].

\subsection{Quadruplexes (GQ)}

\section{$<$ insert here Figure 4>}

G-quadruplexes (GQ) are motifs formed in DNA and/or RNA when four guanine bases associate through Hoogsteen hydrogen bonding, forming a planar G-quartet (Figure 3D). Two or more quartets subsequently stack onto one another resulting in a helical conformation (Figure 4) [182].

Several computational studies have shown their prevalence in the human genome $[183,184]$ and transcriptome [185]. Most interestingly, these well-conserved sequences are stable under physiological conditions and are mostly found in regulatory regions of DNA [89] or RNA [90], hinting at possible roles in regulating cellular processes.

The differences in linking sequences induce conformational heterogeneity, as do other aspects such as nucleotide sequence, temperature, concentration, and the type of cation present [186]. This makes structural predictions very challenging for GQs. Both DNA and RNA GQs form either intermolecular or intramolecular motifs. However, RNA GQs are more stable due to better stacking of the quartets and additional intramolecular hydrogen bonds with the 2'-OH group [187]. Because of the preferred anti conformation of the ribose sugar RNA GQs are always parallelstranded [188] thus having less conformational heterogeneity than the corresponding DNA sequences.

Single-molecule FRET is useful to study the conformational heterogeneity and real-time dynamics of G-rich DNA sequences folding into intramolecular quadruplex structures $[189,190]$. Such studies on G-rich RNAs are missing yet. As a general strategy, a 5' acceptor-labeled GQ forming sequence is followed by a duplex overhang containing the donor fluorophore (acceptor, Cy5; donor, TMR or Cy3) [191]. In some cases, one of the two strands is biotinylated at the 3'-end for surface immobilization.

The first smFRET experiments with the telomeric GQ-forming sequence (htelo) showed two interconverting FRET states in both $\mathrm{Na}^{+}$and $\mathrm{K}^{+}$solution: A low-FRET state at 0.35 (at $10 \mathrm{mM}$ salt) or 0.52 (at $100 \mathrm{mM}$ salt) and a high-FRET state at 0.85 (independent of salt concentration) [191]. Heating of a sample in $10 \mathrm{mM} \mathrm{NaCl}$ reduced considerably the high-FRET population, which increases again upon addition of $10 \mathrm{mM} \mathrm{KCl}$. This change in quadruplex stability depending on 
the monovalent ions present agrees with UV melting measurements and with the widely reported GQ stabilization by $\mathrm{K}^{+}$[192].

With a similar htelo construct, three different cation- and temperature-dependent FRET states were observed at $2 \mathrm{mM} \mathrm{K}^{+}$: Low $(\sim 0.3$, ssDNA), intermediate $(\sim 0.6$, parallel GQ) and high $(\sim 0.8$, antiparallel GQ) [193]. The low-FRET conformation was the only one appearing in the absence of monovalent ions, and its population decreased as the $\mathrm{K}^{+}$concentration increased. Higher concentrations of $\mathrm{Na}^{+}$compared to $\mathrm{K}^{+}$were required for the same effect. As expected, not only the concentration but also the nature of the monovalent cation had an effect on the distribution of GQ conformations. Another example was observed for the $O$. nova GQ when comparing $\mathrm{K}^{+}, \mathrm{Na}^{+}$and $\mathrm{Li}^{+}$[194]. At $10 \mathrm{mM} \mathrm{K}^{+}$, three distinct FRET peaks were observed in single molecule histograms at 0.2 (low, ssDNA), 0.5 (middle, parallel GQ) and 0.76 (high, antiparallel GQ). This polymorphism was also seen in CD spectra at $200 \mathrm{mM} \mathrm{K}^{+}$where there was an overlap of the parallel and antiparallel characteristic peaks. In contrast, an antiparallel-only fold was observed in presence of $\mathrm{Na}^{+}$, as seen by CD (at $200 \mathrm{mM}$ ) or smFRET $(100 \mathrm{mM}, 0.65$ FRET). In the presence of $10 \mathrm{mM} \mathrm{Li}^{+}$only one peak appeared at $0.2 \mathrm{FRET}$, a second peak arising at 0.75 at $30 \mathrm{mM} \mathrm{Li}^{+}$, and a third one at 0.64 at $300 \mathrm{mM} \mathrm{Li}$. The unfolding rates in the presence of $\mathrm{Li}^{+}$are approximately 1000 -fold higher than in the presence of $\mathrm{K}^{+}$. That means the folded conformation is altered depending on the surrounding cation environment.

On the other hand, comparison of single molecule results for the O. nova GQ with those measured under ensemble fluorescence conditions showed that the observed increase of Cy5 fluorescence upon $\mathrm{K}^{+}$addition was due to the population increase of higher FRET conformations [194]. Indeed, the low-FRET peak is highly dominant at low $\mathrm{K}^{+}$concentrations, disappearing at high concentrations; while the middle and high peaks are not visible at $0.1 \mathrm{mM} \mathrm{K}$, and gradually appeared as the concentration was increased. For the above-described study of htelo GQ at $2 \mathrm{mM}$ $\mathrm{K}^{+}$, kinetic bulk FRET measurements suggested the existence of multiple states with different lifetimes, agreeing with the long-lived and short-lived species observed in single molecule experiments [193]. Under physiological conditions $\left(37^{\circ} \mathrm{C}, 140 \mathrm{mM} \mathrm{K}{ }^{+}\right)$folded htelo is present with comparable populations of parallel and antiparallel conformations [193]. In order to assess the role of htelo in regulating telomere length in vivo, the study of the dynamics and equilibrium between these two conformations is thus important. This concerns especially the interaction with proteins or small molecules.[195] Similarly, the GQ-forming sequences from human promoters 
are being researched as possible drug targets for future anticancer strategies [196]. A natural $c$ $M Y C$ sequence from the promoter of the corresponding gene showed a kinetically stable medium FRET subpopulation at $\sim 0.6$ FRET, which unfolds roughly 100-fold slower than the at $\sim 0.9$ highFRET population upon complementary strand addition) [190]. This could be of biological relevance and have a role in $c-M Y C$ oncogene silencing [197].

Different constructs and sequences from the native human c-kit promoter have also been studied. In $100 \mathrm{mM} \mathrm{K}^{+}$, kit-1 showed a low-FRET species ( 0.2, unfolded form), a medium-FRET ( 0.5) and a high-FRET ( 0.7), all also observable in the absence of $\mathrm{K}^{+}$but in the presence of $100 \mathrm{mM}$ $\mathrm{Li}^{+}$[198]. kit-2, an equivalent single-stranded construct, had a greater structural heterogeneity with one unfolded and three folded structures in $100 \mathrm{mM} \mathrm{K}^{+}(\sim 0.2, \sim 0.4, \sim 0.7$, and $\sim 0.85)$. The influence of $\mathrm{K}^{+}$was more pronounced in the kit-2 versus the natural duplex environment in kit-1. Less than $1 \%$ of the molecules of both constructs showed dynamic fluctuations during an observation time of $30 \mathrm{~min}$ (with encapsulation in immobilized vesicles) [199]. This lack of dynamics contrasts with htelo and suggests that the dynamic behavior of intramolecular DNA GQs would be intrinsic to every sequence. In a second study, two different GQ-forming sequences from c-kit formed parallel GQs by CD [200]. Both presented only one high-FRET species $(\sim 0.9)$ in $100 \mathrm{mM} \mathrm{K}^{+}$, which decreased in the absence of $\mathrm{K}^{+}$. In $100 \mathrm{mM} \mathrm{Na}^{+}$, additional medium- and high-FRET states were observed. Interestingly, the calculated half-life of both GQs (of the order of $10^{4} \mathrm{~s}$ ) would be long enough to allow interaction with small molecules.

Recently, the first study on the interaction of a protein with a GQ was reported $\left(150 \mathrm{mM} \mathrm{Na}^{+}\right)$by Hwang et al. [201]. Four steps of FRET decrease $(0.8>0.6>0.45>0.3>0.2)$ indicate a sequential unfolding of the htelo GQ ([TTAGGG $\left.]_{4}\right)$ by two POT1 protein monomers [202]. Upon addition of POT1-TPP1N [203] a fluctuation at mid- to low-FRET implied a continuous unfolding and refolding of the GQ assigned to a sliding motion of the complex, which could be biologically important for telomerase loading and propulsion.

\section{Single molecule movie, image and signal processing}

The single molecule investigation on the influence of metal ions on RNAs as summarized in Section 2 require a vast amount of measurements, often titration studies, and subsequently a very tedious, time consuming, and rigorous data treatment and evaluation. In addition, several aspect of the influence of metal ions on the photophysical properties of the fluorophores need to be taken 
into account. Importantly, the heterogeneity observed as a variety of FRET states, their population, and their transition rates were found to be a metal ion dependent feature. Hence, also the determination of the sm heterogeneity on a quantitative basis with the help of statistical approaches is aimed for [204]. There is a variety of solutions for different analyzing steps, like the background correction or the determination of FRET states, however, a quantitative comparison of those is missing, but will be addressed elsewhere [205]. In this section we summarize the most important specific aspects of smFRET, when performing such experiments.

In fluorescence based single-molecule techniques, the fluorophores are exposed to a high excitation photon flux and are thus subject to photophysical and photochemical phenomena, observable as luminescence intermittence (also known as photo-blinking) and/or photo-bleaching $[29,206]$. The finite number of photons before bleaching enforces a compromise between time resolution and total observation time. Usually, a lower excitation power is favored to ensure longer observation times, which yield a lower signal-to-noise ratio (SNR) leading to a challenging data analysis. The range of FRET simulation and analysis software tools for single-molecule applications is broad [73][5,207][24,208] and will be addressed below to give an overview of preand post-processing steps for the (fluorescence) intensity time trace generation of camera based smFRET measurements. This workflow has been designed considering the wide variety of photophysics and the dynamic heterogeneity within a set of molecules in a sm sample.

\subsection{A guide for smFRET analysis}

Using our recent smFRET studies on the metal ion-dependent folding of the group IIB intron ribozyme Sc.ai5 $\gamma$ [22,75] (Figure 5 and Figure 6) and the 5'-splice site formation EBS1-IBS1 [74], i.e. an RNA-RNA docking process (Figure 1B and Figure 7), as examples, we here guide through the analyzing steps for smFRET data, highlighting the crucial points for evaluation and interpretation of metal ion-induced heterogeneity. All experiments were carried out in a homebuilt TIR fluorescence microscope described elsewhere [209].

\section{$<$ insert here Figure 5>}

The well-studied group IIB intron derivative $S c$.D135 was modified for the use in smFRET studies with the insertion of two loops (L) in domains D1 and D4 and an elongated 3'-tail. The derived construct D135-L14 is depicted in Figure 6. The fluorescent labeling was performed by hybridizing Cy3- and Cy5-carrying DNA oligonucleotides with loops 1 and 4. The 3'-end 
elongation was hybridized with a biotin-DNA for surface immobilization via biotin-streptavidin binding.

The FRET pair Cy3 and Cy5 was covalently bound to the 5'-ends of the modified exon binding site-containing hairpin $\mathrm{d}^{\prime} \mathrm{EBS} 1 *$ (abbreviated as EBS1*) and the seven nucleotide intron binding site $\mathrm{IBS}^{*}$, respectively (Figure 7) [50]. EBS1* was further extended with a 3'-poly-U tail carrying a biotin for immobilization. The IBS $1 *$ cognate is allowed to freely diffuse in solution in appropriate concentrations that allow for sufficient binding. Stabilization of the EBS1*-IBS1* complex is facilitated by Watson-Crick base pairing in the presence of divalent metal ions, in particular $\mathrm{Mg}^{2+}[74]$.

\subsection{Background correction}

To obtain background-free fluorescence intensity time traces of each single emitter the redundant background signal must be determined. This is an inherent feature of sm video microscopy due to the read-out and/or thermal noise of the (EM)CCD camera and additional noise sources like Raman scatter of the excitation field, sample auto-fluorescence, background fluorescence of freely diffusing fluorophores etc. [210]. Ideally, microscopes for sm detection and the sm sample preparation protocol are designed to reduce, or even to avoid, most of the background sources. Still, the remaining intrinsic noise of the camera must be corrected. In the case of homogeneous background such as the camera noise, the noise distribution is approximated by a Gaussian function (as converging limit of the Poisson distribution for photon emission) and can be corrected by subtracting its mean value from the recorded sm image or the subsequently generated time traces. The global background correction is available within the camera software either by recording the background of a blank sample and subtracting the background from all frames or by applying image filters to each frame after the movie is recorded (Figure 5A-1/2).

In most experiments the background is heterogeneously distributed, e.g. the pattern of the excitation light beam in prism-based TIRFM or the individual molecule environment, and is therefore dependent on the molecules' position. Consequently, the background needs to be defined for each individual molecule either by determining the local background represented by the average intensity of a number of molecule-free area or by the remaining intensity signal after single-step photobleaching [101].

Figure 5A exemplifies the reduction of our slightly inhomogeneous background by applying a 
filter published by Crocker and Grier for digitized video microscope images of colloidal suspensions (Figure 5A-3) [211].

\section{3 sm spot detection}

Single-molecule localization [212], also denoted as spot detection in fluorescence microscopy, is based on the sm intensity profile - the so-called Point Spread Function (PSF) - usually approximated by a Gaussian function [213]. The PSF covers a number of pixels on the CCD camera and appears as an intensity spot with circular symmetry. In the simplest approach these spots are chosen manually, e.g. with the help of ImageJ (Image Processing and Analysis in Java) [214]. Efficient, specialized, and fast algorithms for automated spot detection have been developed in the field of single-molecule diffusion [215-217], and for optical super-resolution techniques, such as PALM, STORM, and variants [218-222]. The image-processing toolbox of Matlab (Mathworks) offers a fast spot detection by using the houghpeaks algorithm, which allows the user to consider spots according to their dimensions and intensity. The determined spot coordinates for an example are highlighted by circles in Figure 5A-4. For a precise localization, spot centers are determined by centroid calculation or Gaussian fitting. The shape of the Gaussian represented by the width and amplitude may be used to discriminate a single-molecule from a superposition of several molecules, since overlapping PSFs yield asymmetric spot profiles.

\subsection{Co-localization and time trace generation in multi-channel experiments}

sm techniques split the field of view (FOV) into multiple parts, which contain spectral or polarization information [6,223-225]. The incoming light beam is separated by a dichroic mirror or a polarizing beam splitter. The resulting sub-images are directed and focused on to the camera detector. For multiple channels, the localization of the same molecule appearing throughout is required. The determination of the associated coordinates of one individual molecule is referred to as co-localization. To perform this co-localization, usually fluorescent beads are immobilized on a cover slip surface as they are bright and easy to link for different spectral channels. A general rule applies that the more and more evenly distributed spots are co-localized, the better the transformation/co-localization function will be. The set of input coordinates is the basis of the transformation matrix calculated with the co-localization algorithm, e.g. a local weighted mean transformation (Figure 5A-4).

To avoid the loss of molecules with low intensity the spot detection for multi-channel video 
microscopy is processed separately in each channel. After applying the co-localization or coordinate transformation to obtain the coordinates in the respective channels single molecules may occur multiple times. Thus a proximity criterion is used to exclude multiplicities (Figure 5A5).

The subsequent generation of time traces after determination of the $\mathrm{sm}$ coordinates is straightforward. Usually, the number of pixels covered by the PSF are integrated or just summed. This procedure may be further restricted by considering only those pixels which improve the SNR. Associated fluorescence intensity time traces are created from the co-localized coordinates and used to calculate physical measures like the FRET efficiency [3].

A set of time traces shown in Figure 5B exemplifying conformational transitions of D135L14 and photobleaching was derived from integrating over $3 \times 3$ pixels. The green and the red trajectories represent the emissions originating from the donor/acceptor dyes (Cy3/Cy5). The background of the acceptor signal can easily be determined from the remaining signal after bleaching. The background for the donor is determined from a neighboring area showing no spots. The ratio of $\mathrm{Cy} 5$ to the sum of $\mathrm{Cy} 3$ and $\mathrm{Cy} 5$ emission intensities yields the apparent FRET value. The typical FRET histogram of a single trace with and without the remaining part of the time trace after photobleaching is presented in Figure 5D.

\section{5 sm exclusion rules}

sm analysis is usually semi-automated since only particular trajectories may be considered for further analysis. There is no general rule on how to identify such molecules. To distinguish between molecules of interest and fluorescent contamination it is recommended to investigate the solvent/buffer or the biomolecule without the fluorophore labels under the same experimental conditions. In some cases, sm time traces show a very erratic or a low intensity signal and are thus easily excluded. Photobleaching is unavoidable with organic fluorophores and numerically detectable by a thresholding process of the intensity traces. Photo-bleached parts of the trajectory bias FRET and dwell-time analysis and must hence be eliminated from further analysis (Figure 5).

Spot exclusion is necessary if two or more PSFs overlap. However, the separation of two overlapping PSFs depends of intensity and distance and is thus not applicable in all cases. The respective traces may either exhibit more discrete states or increased intensity, resulting in multi- 
step photobleaching. To identify such artifacts measurement times are extended to bleach all labels of one molecule. Increasing the average distance between the molecules decreases the probability of indistinguishable PSF overlap. Both methods have to be adapted to the individual experimental conditions.

\subsection{Reducing noise in sm time traces}

A method to reduce noise in stochastic trajectories of single molecules applied to single proteins was published by Gilad Haran [226], who generalized the non-linear filtration technique developed by Chung and Kennedy [227]. The Haran filter reduces the noise in single-molecule trajectories while retaining sharp features and thus facilitating the detection of conformational transition states in a noisy trajectory. Furthermore, it also provides better estimates for the temporal positions of jumps between states and dwell-times than a standard low-pass filter. As a disadvantage, the bandwidth is reduced in stretches of data that do not contain sharp state-to-state transitions.

Another approach to reduce the noise is the singe molecule trajectory wavelet analysis and Bayesian Inference [228-230]. The method distinguishes photoblinking from large shifts in the smFRET efficiency and is validated on simulated and experimental data retaining their underlying dynamic properties.

\section{7 $\quad$ Alternating laser excitation (ALEX)}

This technique [17] is used for TIRF-based video microscopy of single immobilized molecules to achieve fluorescence-aided molecular sorting (FAMS) [231]. Figure 5B shows the donor and acceptor fluorescence intensity trajectory of a Cy3/Cy5-labeled group IIB intron derivative D135L14 after green excitation at $532 \mathrm{~nm}$ as well as the control signal of the acceptor after red excitation at $640 \mathrm{~nm}$, respectively. The frame rate was chosen to be $10 \mathrm{fps}$, giving $100 \mathrm{~ms}$ integration time for each spectral channel. Thus, the overall time resolution was $200 \mathrm{~ms}$. the trajectory of the apparent FRET and the stoichiometry $S$ are shown below (Figure 5C). The stoichiometry is plotted versus the apparent FRET yielding a density plot representation (Figure 5D). This allows to distinguish four different cases: (i) Bleaching of the acceptor, or (ii) donoronly labeled molecules, both located around the FRET-S pair $(0 ; 1)$; (iii) acceptor-only labeled molecules are distributed around $S \sim 0$ and show a broad FRET distribution due to shot noise; (iv) acceptor blinking is visible as broadened $S$ distribution. In the latter case, the absolute FRET 
values broaden due to unusual control signal fluctuations of the acceptor. Photophysical or anisotropic effects of the acceptor fluorophore Cy5 might cause this effect yielding a broadened FRET distribution. At high $S$ values the respective FRET value is generally lower than at lower $S$ values. Therefore, S-FRET density plots serve as fingerprints of individual molecules. In single molecule wide field microscopy, ALEX is of particular interest to distinguish between a "real" zero FRET state and donor-only labeled species.

For the example trace in Figure 5, the acceptor intensity during red excitation (control signal) remains high till the acceptor photobleaches, leading to a step in the donor intensity. However, observed intensity fluctuations of the dyes in single molecule experiments may have (beside FRET) either a photophysical or photo-chemical background $[29,206]$ or emerge from fluorophore re-orientation or anisotropic effects [3,223,232], in particular near surfaces [233], herein visible as intensity fluctuation of the donor signal after acceptor bleaching. For control experiments it is of importance to characterize the donor-only and acceptor-only labeled fluorescence trajectories separately and to characterize the fluorophores attached to the NA by means of fluorescence lifetime and dynamic anisotropy measurements, to ensure free dye rotation (compare AV calculation of the fluorophores attached to EBS1*-IBS1* in Figure 7A [234]).

\subsection{Static and dynamic single-molecules}

Due to the limited observation time and/or too high activation barriers individual molecules might be observed not to undergo any transitions and will therefore be denoted as static molecules. This has been observed for the 5 -splice site formation of EBS1*-IBS1* [74]. The fractions of static EBS1* molecules that are either unbound (low FRET) or bound to IBS1* (high FRET) during the total observation time (400s) depend strongly on the $\mathrm{Mg}^{2+}$ concentration. An increase of the fraction of completely bound molecules upon increase from 1 to $8 \mathrm{mM} \mathrm{Mg}^{2+}$ was found. These subpopulations are attributed to a higher binding affinity. Another subpopulation remains completely unbound and has therefore a much lower binding affinity. The quasi-ensemble binding constant hides these heterogeneities. Nonetheless, it was verified that the single molecule average is still consistent with the respective bulk studies.

In Figure 6B dynamic and (mostly) static molecules depending on the $\mathrm{Mg}^{2+}$ concentration for the group IIB intron derivative D135L14 are shown [76]. Amongst the static molecules the unfolded state is less frequent while the number of more compact structures like the intermediate conformation I increases upon increasing the $\mathrm{Mg}^{2+}$ concentration from $1 \mathrm{mM}$ to $100 \mathrm{mM}$ [75]. 


\section{9 $\quad$ Modelling the correct number of states}

To find the overall number of accessible molecular conformations, the FRET histograms of all single molecules considered for analysis are summed up and fitted by a sum of Gaussians [235]. The number of Gaussians can be determined by fitting the histogram with an increasing number of Gaussians reducing the fitting error (compare Figure 6A). Whether the full width at half maximum (FWHM) or the number of Gaussians fitted to a distribution is varied is often defined by a user-dependent model. However, free fits and statistical tests are combined to obtain more objective conclusions about the suitable number of states. Herschlag et al. presented a software package that offers statistical tests and uses a Bayesian Information Criterion (BIC) as objective measure to validate the significance of a certain number of states [208]. In multivariate systems, e.g. FRET distributions of multiple FRET states, it is particularly difficult to achieve reliable fitting results. To get access to a number of FRET states and their population under different experimental conditions e.g. by varying the metal ion concentration, global fitting routines are applied. The same fit model, which is well motivated by the system under study and defined by a set of fitting parameters, is applied to different data sets at the same time. By cross-linking the fitting parameters between different sets of data, the number of free parameters is dramatically reduced, thus, the fitting error can be decreased by decreasing the degree of freedom of the fit model [236]. Further, global fitting allows to distinguish whether shifts of FRET states are due to conformational changes under different experimental conditions like in metal ion titration experiments or due to cross-sample variability etc. [24].

The quantification of the transition dynamics of tens to hundreds of molecules at the same time over a large range of observation times is one of the general advantages of sm video microscopy. Two methods are used to determine transition rates, the dwell-time analysis and the auto- or crosscorrelation of individual time traces. Both methods are generally applicable to noisy trajectories. The dwell-time analysis requires the discretization of the noisy signal into intervals of quasiconstant intensities (dwell-times). Here, the accuracy depends on the SNR, the absolute change of the intensity signal between two successive states, and the absolute rate of interconversion. At a low SNR and with particular small changes in the signal intensity the state determination becomes erroneous. Sophisticated methods for the state determination in noisy intensity time traces based on Hidden Markov models (HMM) [237] have been reviewed by Nils Walter [238]. A comparison of threshold-based [239] and Hidden Markov Model based dwell-time analysis is presented 
elsewhere [208]. In particular, HMM based methods show a higher accuracy at lower SNR. An elected number of software tools based on HMM algorithms for the purpose of single molecule trajectories discretization are HaMMy [139], vbFRET [240], QuB [241,242], and SMART [208] as well as a HMM toolbox from Kevin Murphy modified by Pirchi et al. [243]. We use vbFRET, and apply the most suitable model by selecting the lowest BIC (Bayesian Inference Criterion) [240].

Hyeon et al. studied the dynamics of DNA holiday junction as a simple two-state system in presence of $\mathrm{Mg}^{2+}$ [21]. Interestingly, both the cumulative dwell time and the sum FRET histogram do not exhibit heterogeneity and the system is well described as two-state system with a single exponential decay seemingly ergodic (the time average of each single molecule converges to the ensemble average for unlimited observation times). However, single FRET time traces show differences in the population of both states and truly vary in their individual dwell time distribution characterizing the system as non-ergodic. Applying a K-mean clustering algorithm, the ensemble of single time traces is parted into $K=5$ clusters, ensuring that each cluster's dynamics is ergodic. The rates vary to a large extend between the five ergodic subspecies and are attributed to the formation of internal multi-loops at the junction. This study showed the potential of clustering to elucidate the samples heterogeneity of NA.

However, if the kinetics are fast and close to the time resolution of the camera state transitions will be of continuous nature, i.e. no discrete states can be observed, or the intensity changes are close to the signal fluctuations correlation methods are applied to the trajectories. The respective kinetic rates are thus obtained by fitting of a model function to the correlated data [107,109]. Another elaborated technique to detect intensity change-points was published by Yang et al. They use a generalized likelihood ratio test to determine the location of an intensity change point based on individual photon arrival times accessible by means of time-tagged single photon counting (TCSPC) measurements [244-248]. Combining change-point analysis (CPA) with clustering methods allows the discrimination of a finite number of states even in strongly fluctuating signal trajectories [248]. The CPA method circumvents time binning (photons into groups) in camerabased video microscopy and might give further details about averaged or hidden kinetics especially when the aforementioned methods are limited.

The time-dependent FRET fluctuations shown in Figure 6 stem from shot noise and folding or conformational dynamics of the group II intron. FRET trajectories are thus analyzed by means of 
HMM using vbFRET [240]. The number of states is derived by making use of a BIC [240]. In total four states $(0.1,0.21,0.36,0.56)$ are attributed to the unfolded, the intermediate I and II as well as the native state of the group II intron. Figure 6 exemplifies time traces at different $\mathrm{Mg}^{2+}$ concentrations. At low $\mathrm{Mg}^{2+}$ concentration the most compact state $(F R E T \cong 0.56)$, which is supposed to be the native conformation, is only rarely populated. In contrast, higher $\mathrm{Mg}^{2+}$ concentrations are necessary to allow the RNA to transiently reach its native state. Upon addition of $\mathrm{Mg}^{2+}$ from $1 \mathrm{mM}$ to $100 \mathrm{mM}$ the more compact states are increasingly stabilized, exemplarily shown by the increased occurrence of high FRET states in the presence of higher $\mathrm{Mg}^{2+}$ concentrations. Still, the total number of states of such large RNAs is also associated with the experimental conditions as shown in smFRET studies in a highly crowded environment [76].

\subsection{0 sm kinetics}

Dwell-times have to be assigned (i) to a specific state and/or (ii) sorted respective to their next transition state. This is less problematic if the occurring FRET states are well separated, but becomes challenging in case of close-lying FRET states, a low SNR, or heterogeneous broadening due to instrumental sources, etc. [139]. To overcome these issues all observed FRET values before and after a detected transition are used to build a 2D transition density plot (TDP). Accumulating transitions, i.e. from state $\mathrm{n}$ to $\mathrm{m}$, are identified as clusters, e.g. of a spherical or an elliptical shape $[105,138,139]$. Which signatures in the TDP belong to the same transition is defined by the user, but can be automated by clustering analysis [105]. After discretization of single molecule trajectories and TDP clustering, dwell-time histograms (or their cumulative variant) and probability density plots $p\left(t_{\mathrm{dwell}}\right)$ of the respective transitions are fitted by an exponential decay model to determine kinetic rate constants [141]. The amplitudes of the respective exponential time constants are directly related to their occurrence. In the case of hidden states/transitions, other fit functions are required [249]. Hellriegel et al. follow a different approach and determine the cumulative probability distribution $P($ Var $)$ by sorting the dwell-times by ascending order [250]. As outlined above, single exponentials for homogenous and multi- or stretched-exponential functions for heterogeneous systems are applied to the (cumulative) probability distributions, while gamma distributions are applied if hidden states are assumed to exist [249,251]. Note that single molecules do not contribute equally to such dwell-time distributions due to the finite observation time. An elevated number of dwell-times will be included in the dwell-time histogram for molecules of high transition rates compared to those showing only few transitions in the same 
detection period. This is in particular of relevance when comparing thermodynamics and kinetics, as state transitions with a low rate are naturally underrepresented in both, the dwell-time histograms and the TDPs, but the associated states can still predominantly occur in the FRET time traces and the respective FRET histograms.

\section{<insert here Figure 6>}

In the case of the group II intron, at least four Gaussians are necessary to fit the overall FRET distribution. The transition frequency is an information not contained in the FRET histograms but obtained from the discretized dwell-time trajectories. The TDP for the group II intron shows transitions between four populated FRET states. Furthermore, not only stepwise-transitions, also called on-axis, between neighboring states occur, but also direct transitions, also known as offaxis, e.g. from the lowest (0.1) to the highest (0.6) FRET state (Figure 6D). Whether the intermediate FRET states for such off-axis transitions are only transiently populated or hidden due to the limited time resolution $(100 \mathrm{~ms})$ remains unclear. Different methods like the probability distribution analysis (PDA) [26,252,253] or stroboscopic excitation schemes for ALEX, short sALEX [254], have been proposed to distinguish hidden or transient states from direct transitions between non-neighboring states and FRET broadening due to shot noise. SALEX in particular increases the time resolution in camera-based detection, enabling to detect faster dynamics than the frame rate of the camera.

Upon addition of $\mathrm{Mg}^{2+}$ from $1 \mathrm{mM}$ to $100 \mathrm{mM}$ the more compact states are increasingly stabilized, exemplarily shown by the increased occurrence of high FRET states in the presence of larger $\mathrm{Mg}^{2+}$ concentration as shown in Figure 6. The fluctuations between two states are fast and the absolute difference between the FRET states is low making the dwell-time analysis challenging (compare to Figure 6D). Thus, the number of transitions can only be assigned in individual trajectories rather than in the TDP. The number of known tertiary contact interactions of the group II intron is manifold $\left(\alpha-\alpha^{\prime}, \beta-\beta^{\prime}, \kappa-\mathrm{k}^{\prime}, \theta-\theta^{\prime}, \lambda-\lambda^{\prime}, \kappa-\zeta\right)$. Hence, $\mathrm{Mg}^{2+}$ ion-dependent TDP provide useful insights to the occurring folding pathways and support that (i) $\mathrm{Mg}^{2+}$ facilitates the transitions towards the active state, (ii) these transitions occur on a milliseconds to seconds time scale and (iii) the active state is only transiently occupied showing less structural variety than the extended conformation.

\subsection{Bootstrapping to access sample variability}




\section{<insert here Figure 7>}

In camera-based detection a sufficient number of single molecule time traces, i.e. the sample distribution, is expected to reflect the whole molecule ensemble (ergodicity). Different molecules in the same conformational state might appear at slightly different FRET values due to heterogeneous broadening or sample heterogeneity. This effect depends on the experimental conditions, the accuracy of image/signal post-processing, and the photophysical aspects [139]. To get access to both the sample heterogeneity as well as the cross-sample variability, König et al. applied a bootstrapping algorithm to smFRET data [204]. Bootstrapping relies on the statistical sampling of the sample distribution. $N$ values of the sample of size $n$ are randomly chosen with an equal probability of $1 / n$ and multiple selections are allowed. The resampling is performed $M$ times, typically $M=100$, to access the sample variance. Bootstrapping was applied to both, the FRET histogram to render the variance of thermodynamics, i.e. the population of FRET states

(Figure 7B), and the cumulative dwell-time histograms (Figure 7C) to characterize the variance of kinetics, i.e. the rate constant for transitions between FRET states. Subsequently, statistical hypothesis testing using generalized analysis of variance (ANOVA) can be performed to show whether changes of the population of FRET states or their transitions are statistically significant [257].

For EBS1*-IBS1* the stabilization effect of $8 \mathrm{mM}$ compared to the absence of $\mathrm{Mg}^{2+}$ is exemplified in Figure 7B [74]. The FRET histogram shows a pronounced increase of the high FRET state representing the stabilization of RNA-RNA docking in the presence of divalent metal ions. Importantly, also trace amounts of $\mathrm{M}^{2+}$ ions already present in puriss p.a. monovalent salt, induce RNA-RNA docking [74]. Bootstrapping showed that the variability of the state populations indeed increases in the presence of $\mathrm{Mg}^{2+}$, but the change in the population remains statistically significant. This alleviation of the 5'-splice site recognition upon increase of $\mathrm{Mg}^{2+}$ concentration is assigned to stabilize the catalytically active conformation of the group IIB intron observed in $\mathrm{Mg}^{2+}$ titration experiments. The considerably higher population of the bound state in the presence of 8 $\mathrm{mM} \mathrm{Mg}{ }^{2+}$ is caused by the 1.5 orders of magnitude higher binding affinity compared to the absence of $\mathrm{Mg}^{2+}$. In NMR experiments of the same construct metal ion binding sites were identified close to the RNA duplex formed by EBS1 and IBS1 (compare Figure 1B), showing affinities in the millimolar range [50,74]. In that range, the strongest stabilization effect was observed in smFRET experiments for the EBS1*-IBS1* interaction too [74]. $\mathrm{Mg}^{2+}$ ions in 
crystallized group II introns from Oceanobacillus iheyensis are close to exon-intron binding sites $[50,258]$, supporting the discussion above.

For the EBS1*-IBS1* interaction, the TDP is rather trivial as only two states are populated (the docked and undocked state) [74]. The increased stabilization of EBS1*-IBS1* due to an increase of $\mathrm{Mg}^{2+}$ arises not only from increasing docking rates but also from decreasing dissociation rates, thus increasing decay times, as shown in the complementary cumulative dwell-time histograms derived from threshold-based dwell-time analysis performed subsequently to TDP clustering (Figure 7C). The cumulative dwell-time histograms in the presence of $\mathrm{Mg}^{2+}$ were only suitably fitted by two or three exponentials with (dwell) time constants ranging from sub-seconds to several tens of seconds ascribing kinetic heterogeneity. Using bootstrapping allows to access the variability of the rate constants. In particular the long decay times in the range of tens of seconds show a high variability proofing differences of the lower docking and undocking rates to be statistically not significant. The authors showed that the observed kinetic heterogeneity is most pronounced if the $\mathrm{Mg}^{2+}$ binding sites are half occupied, i.e. between 1 and $8 \mathrm{mM} \mathrm{Mg}{ }^{2+}$. They further concluded that the observed heterogeneity of the RNA-RNA docking/undocking process is due to a structural permutation in the case of incomplete occupation of the $\mathrm{Mg}^{2+}$ binding sites [74]. Interestingly, the kinetic heterogeneity vanished in the case of co-encapsulated EBS1* IBS1* molecules at $50 \mathrm{mM} \mathrm{Mg}^{2+}$ in surface immobilized lipid vesicles. Thus, the close proximity of the cognates and the high effective metal ion concentration in the vesicles leads to a complete occupation of the $\mathrm{Mg}^{2+}$ binding sites. Further, smFRET $\mathrm{Mg}^{2+}$ pulse experiments showed that most of the d3'EBS1*-IBS1* complexes remain stable other seconds after $\mathrm{Mg}^{2+}$ depletion by adding EDTA; after re-addition of $\mathrm{Mg}^{2+}$ the docking of IBS1* occurred with a delay in the range of seconds [74]. Thus, the $\mathrm{Mg}^{2+}$ exchange rate is shifted to a range of $10^{-2}$ to $10^{2} \mathrm{~s}^{-1}$ [74], extending previous predictions for $\mathrm{M}^{2+}$ expected to exchange in the range of milliseconds [62].

\section{Conclusions}

Understanding how cofactors like metal ions influence nucleic acids in terms of structure stabilization and re-organization is essential to understand their function in vivo. Fluorescencebased single-molecule techniques like single molecule Förster resonance energy transfer applied on nucleic acids in vitro have been summarized herein, with a particular focus on the role of metal ions. An intriguing commonality is the observed kinetic and structural heterogeneity underlying 
most of the presented RNA and DNA structures, but explanations are rare. The correlation between RNA structure, metal ion concentration, and kinetic heterogeneity observed in small RNA structures suggested to analyze the heterogeneity sensitive to metal ions in more complex RNAs, such as ribozymes and riboswitches, yielding similar results. Transiently populated states that interconvert during the observation time have been reported by Lee et al. to explain the observed kinetic heterogeneity in NA [259]. Both, the specific and the unspecific interaction of metal ions with NAs play a major role in RNA folding and function and are thus supposed to be responsible for the sm heterogeneity. Recently, Kowerko et al. provide first experimental evidence that kinetic heterogeneity in RNA folding and interaction stem from incomplete occupation of divalent metal ion binding sites [74]. The binding affinity of $\mathrm{Mg}^{2+}$ to their binding sites in NA are in the millimolar range. At physiological $\mathrm{Mg}^{2+}$ concentration binding sites are not fully occupied, thus, explaining the observed heterogeneity in NA single molecule studies. Inner and outer sphere coordinated $\mathrm{M}^{2+}$ were supposed to exchange on a millisecond time scale [260], but have been found in $\mathrm{Mg}^{2+}$ pulse experiments to be in a range of $10^{-2}$ to $10^{2} \mathrm{~s}^{-1}$, thus observable in camera based detection [74]. Similar heterogeneities of RNA-RNA interactions have been discussed previously, e.g. for the kissing complex $[103,118]$ and the hairpin ribozyme $[107,109]$, leaving the occupation of $\mathrm{M}^{\mathrm{n}+}$ binding sites as a major criterion to understand the single molecule heterogeneity of NAs.

The highly automated, software-supported movie and data processing is fundamental to handle the increasing amount of data in contemporary single-molecule microscopy and camera-based detection. Here we provide an overview of the established methodology and guide the reader through all steps of single molecule movie processing and smFRET data analysis including thermodynamics and kinetics, available in our software package MASH and established holistic frameworks (iSMS, HaMMy, SMART, 2tone etc.). Future comparative studies for sm data analysis will demand to compare existing algorithms (background correction, sm detection and colocalization, time trace generation, the detection of state transitions e.g. with HMM based methods, and the statistical prove of sample heterogeneity) to base the data analysis in sm spectroscopy on default settings for the particular methodology and adapted to induvial experimental conditions.

\section{Acknowledgements}


This work was supported by the European Research Council (ERC Starting Grant MIRNA N ${ }^{\circ}$ 259092, to RKOS) and the Forschungskredit grant of the University of Zurich (FK-14-096 to RB, FK-13-108 to DK, and FK-13-090 to HG). We are very thankful for further financial support related to our smFRET studies by the Swiss National Science Foundation, SystemsX.ch, and the Swiss State Secretariat for Education and Research (COST Action CM1105). Further, we are thankful to Sebastian L.B. König for inspiring Figures 1 and 2 and sharing his enthusiasm in the field of smFRET. We thank Erica Fiorini for providing experimental sm data of the D135L14 presented in Figure 5 and Figure 6. We thank Fabio D. Steffen or providing us with the accessible volume calculation for the Cy3/Cy5 labelled EBS1*-IBS1* system. We thank Melodie C.A.S. Hadzic for uncountable fruitful discussions and the development of the software package MASH. 


\section{References}

[1] W.E. Moerner, L. Kador, Phys. Rev. Lett. 62 (1989) 2535-2538.

[2] Orrit, Bernard, Phys. Rev. Lett. 65 (1990) 2716-2719.

[3] J.R. Lakowicz, Principles of fluorescence spectroscopy, 3rd ed., Springer, New York, 2006.

[4] R.M. Clegg, Curr. Opin. Biotechnol. 6 (1995) 103-110.

[5] P.R. Selvin, T. Ha, Single-molecule techniques: A laboratory manual, Cold Spring Harbor Laboratory Press, Cold Spring Harbor, N.Y., 2008.

[6] T. Ha, T. Enderle, D.S. Chemla, S. Weiss, IEEE J. Sel. Top. Quantum Electron. 2 (1996) $1115-1128$.

[7] T. Ha, T. Enderle, D.F. Ogletree, D.S. Chemla, P.R. Selvin, S. Weiss, Proc. Natl. Acad. Sci. U. S. A. 93 (1996) 6264-6268.

[8] T. Ha, A.Y. Ting, J. Liang, W.B. Caldwell, A.A. Deniz, D.S. Chemla, P.G. Schultz, S. Weiss, Proc. Natl. Acad. Sci. U. S. A. 96 (1999) 893-898.

[9] B. Schuler, E.A. Lipman, W.A. Eaton, Nature 419 (2002) 743-747.

[10] T. Förster, Ann. Phys. 437 (1948) 55-75.

[11] R.M. Ballew, J. Sabelko, M. Gruebele, Nat. Struct. Biol. 3 (1996) 923-926.

[12] S. Rocha, J.A. Hutchison, K. Peneva, A. Herrmann, K. Müllen, M. Skjøt, C.I. Jørgensen, A. Svendsen, De Schryver, Frans C, J. Hofkens, H. Uji-I, ChemPhysChem 10 (2009) 151-161.

[13] M. Gudmand, S. Rocha, N.S. Hatzakis, K. Peneva, K. Müllen, D. Stamou, H. Uji-I, J. Hofkens, T. Bjørnholm, T. Heimburg, Biophys. J. 98 (2010) 1873-1882.

[14] H. Kabata, O. Kurosawa, I. Arai, M. Washizu, S.A. Margarson, R.E. Glass, N. Shimamoto, Science 262 (1993) 1561-1563.

[15] T. Perkins, Quake, D. Smith, S. Chu, Science 264 (1994) 822-826.

[16] E.A. Alemán, R. Lamichhane, D. Rueda, Curr. Opin. Chem. Biol. 12 (2008) 647-654.

[17] A.N. Kapanidis, N.K. Lee, T.A. Laurence, S. Doose, E. Margeat, S. Weiss, Proc. Natl. Acad. Sci. U. S. A. 101 (2004) 8936-8941.

[18] B.K. Müller, E. Zaychikov, C. Bräuchle, D.C. Lamb, Biophys. J. 89 (2005) 3508-3522.

[19] S. Lee, J. Lee, S. Hohng, PLoS One 5 (2010) e12270.

[20] J. Lee, S. Lee, K. Ragunathan, C. Joo, T. Ha, S. Hohng, Angew. Chem. Int. Ed. 49 (2010) 9922-9925. 
[21] C. Hyeon, J. Lee, J. Yoon, S. Hohng, D. Thirumalai, Nat. Chem. 4 (2012) 907-914.

[22] M. Steiner, D. Rueda, R.K.O. Sigel, Angew. Chem. Int. Ed. 48 (2009) 9739-9742.

[23] M. Greenfeld, S.V. Solomatin, D. Herschlag, Biol. Chem. 286 (2011) 19872-19879.

[24] S.J. Holden, S. Uphoff, J. Hohlbein, D. Yadin, L. Le Reste, O.J. Britton, A.N. Kapanidis, Biophys. J. 99 (2010) 3102-3111.

[25] M. Antonik, S. Felekyan, A. Gaiduk, Seidel, C. A. M., J. Phys. Chem. B 110 (2006) 69706978.

[26] E. Nir, X. Michalet, K.M. Hamadani, T.A. Laurence, D. Neuhauser, Y. Kovchegov, S. Weiss, J. Phys. Chem. B 110 (2006) 22103-22124.

[27] H.S. Chung, J.M. Louis, W.A. Eaton, Biophys. J. 98 (2010) 696-706.

[28] S. Kalinin, E. Sisamakis, S.W. Magennis, S. Felekyan, Seidel, C. A. M., J. Phys. Chem. B 114 (2010) 6197-6206.

[29] T. Ha, P. Tinnefeld, Annu. Rev. Phys. Chem. 63 (2012) 595-617.

[30] S. Adhikari, M. Selmke, F. Cichos, Phys. Chem. Chem. Phys. 13 (2011) 1849-1856.

[31] A. Issac, C. von Borczyskowski, F. Cichos, Phys. Rev. B: Condens. Matter 71 (2005) 161302.

[32] J. Schuster, F. Cichos, C. von Borczyskowski, Opt. Spectrosc. 98 (2005) 712-717.

[33] J. Hofkens, T. Vosch, M. Maus, F. Köhn, M. Cotlet, T. Weil, A. Herrmann, K. Müllen, F. de Schryver, Chem. Phys. Lett. 333 (2001) 255-263.

[34] S. Krause, D. Kowerko, R. Börner, C.G. Hübner, C. von Borczyskowski, ChemPhysChem 12 (2011) 303-312.

[35] J.J. Sakon, K.R. Weninger, Nat. Methods 7 (2010) 203-205.

[36] T. Fessl, F. Adamec, T. Polívka, S. Foldynová-Trantírková, F. Vácha, L. Trantírek, Nucleic Acids Res. 40 (2012) e121.

[37] F. Gebauer, M.W. Hentze, Nat. Rev. Mol. Cell Biol. 5 (2004) 827-835.

[38] J.A. Doudna, Nat. Struct. Biol. 7 (2000) 954-956.

[39] J.R. Williamson, Nat. Struct. Biol. 7 (2000) 834-837.

[40] R.J. Jackson, Hellen, Christopher U T, T.V. Pestova, Nat. Rev. Mol. Cell Biol. 11 (2010) $113-127$.

[41] D.M. Lilley, Trends Biochem. Sci. 28 (2003) 495-501.

[42] O. Fedorova, in: J.S. Hartig (Ed.), Ribozymes: Methods and protocols, Humana Press, Totowa, NJ, 2012, pp. 91-111. 
[43] K. Kruger, P.J. Grabowski, A.J. Zaug, J. Sands, D.E. Gottschling, T.R. Cech, Cell 31 (1982) $147-157$.

[44] C. Guerrier-Takada, K. Gardiner, T. Marsh, N. Pace, S. Altman, Cell 35 (1983) 849-857.

[45] S.R. Eddy, Nat. Rev. Genet. 2 (2001) 919-929.

[46] D. Castanotto, J.J. Rossi, Nature 457 (2009) 426-433.

[47] T.M. Henkin, Genes Dev. 22 (2008) 3383-3390.

[48] A. Serganov, D.J. Patel, Nat. Rev. Genet. 8 (2007) 776-790.

[49] E. Freisinger, A. Fernandes, A.P. Grollman, C. Kisker, J. Mol. Biol. 329 (2003) 685-697.

[50] D. Kruschel, M. Skilandat, R.K.O. Sigel, RNA 20 (2014) 295-307.

[51] Accelrys Softwar. Inc., Discovery Studio Visualizer, Accelrys Softwar. Inc., San Diego, 2012.

[52] D.E. Draper, D. Grilley, A.M. Soto, Annu. Rev. Biophys. Biomol. Struct. 34 (2005) 221-243.

[53] M. Pechlaner, R.K.O. Sigel, Met. Ions Life Sci. 10 (2012) 1-42.

[54] P. Auffinger, N. Grover, E. Westhof, Met. Ions Life Sci. 9 (2011) 1-35.

[55] R. Shiman, D.E. Draper, J. Mol. Biol. 302 (2000) 79-91.

[56] S.A. Woodson, Curr. Opin. Chem. Biol. 9 (2005) 104-109.

[57] J. Pan, D. Thirumalai, S.A. Woodson, Proc. Natl. Acad. Sci. U. S. A. 96 (1999) 6149-6154.

[58] V.J. DeRose, Curr. Opin. Struct. Biol. 13 (2003) 317-324.

[59] A. Ramesh, W.C. Winkler, RNA Biol. 7 (2014) 77-83.

[60] J. Schnabl, P. Suter, R.K.O. Sigel, Nucleic Acids Res. 40 (2012) D434-8.

[61] D. Donghi, R.K.O. Sigel, Methods Mol. Biol. 848 (2012) 253-273.

[62] E. Freisinger, R.K.O. Sigel, Coord. Chem. Rev. 251 (2007) 1834-1851.

[63] D. Donghi, S. Johannsen, R.K.O. Sigel, E. Freisinger, CHIMIA 66 (2012) 791-797.

[64] P.P. Zarrinkar, J.R. Williamson, Science 265 (1994) 918-924.

[65] P.P. Zarrinkar, J. Wang, J.R. Williamson, RNA 2 (1996) 564-573.

[66] J. Pan, D. Thirumalai, S.A. Woodson, J. Mol. Biol. 273 (1997) 7-13.

[67] C. Hammann, A. Cooper, D.M. Lilley, Biochemistry 40 (2001) 1423-1429.

[68] A.L. Feig, Biopolymers 87 (2007) 293-301.

[69] S.A. Woodson, Curr. Opin. Chem. Biol. 12 (2008) 667-673.

[70] D. Kruschel, R.K.O. Sigel, J. Inorg. Biochem. 102 (2008) 2147-2154.

[71] L.J. Su, C. Waldsich, A.M. Pyle, Nucleic Acids Res. 33 (2005) 6674-6687.

[72] U. Resch-Genger, M. Grabolle, S. Cavaliere-Jaricot, R. Nitschke, T. Nann, Nat. Methods 5 
(2008) 763-775.

[73] S. Preus, L.M. Wilhelmsson, ChemBioChem 13 (2012) 1990-2001.

[74] D. Kowerko, S.L. König, M. Skilandat, D. Kruschel, M.C. Hadzic, L. Cardo, R.K.O. Sigel, Proc. Natl. Acad. Sci. U. S. A. 112 (2015) 3403-3408.

[75] M. Steiner, K.S. Karunatilaka, R.K.O. Sigel, D. Rueda, Proc. Natl. Acad. Sci. U. S. A. 105 (2008) 13853-13858.

[76] E. Fiorini, R. Börner, R.K.O. Sigel, CHIMIA 69 (2015) 207-212.

[77] B. Alberts, A. Johnson, J. Lewis, Molecular biology of the cell, 5th ed., Garland Science, New York, N.Y., 2008.

[78] J.R. Lorsch, J.W. Szostak, Biochemistry 33 (1994) 973-982.

[79] M.C. Erat, R.K.O. Sigel, Met. Ions Life Sci. 9 (2011) 37-100.

[80] D.E. Draper, Biophys. J. 95 (2008) 5489-5495.

[81] J. Schnabl, R.K.O. Sigel, Curr. Opin. Chem. Biol. 14 (2010) 269-275.

[82] J.C. Bowman, T.K. Lenz, N.V. Hud, L.D. Williams, Curr. Opin. Struct. Biol. 22 (2012) 262272.

[83] R.K. Sigel, H. Sigel, in: J. Reedijk, K.R. Poeppelmeier, T. Chivers, E.V. Antipov, A.M. Abakumov, A.V. Shevel'kov, V.L. Pecoraro, T.W. Hambley, P. O'Brien, N. Revaprasadu, S. Kitagawa, R.L. Bedard, L. Casella, R. Schlögl, J.W. Niemantsverdriet, V.W.W. Yam, S. Álvarez (Eds.), Comprehensive inorganic chemistry II: From elements to applications, Elsevier, Amsterdam, 2013, pp. 623-660.

[84] R.K.O. Sigel, A.M. Pyle, Chem. Rev. 107 (2007) 97-113.

[85] N.A. Denesyuk, D. Thirumalai, Nat. Chem. 7 (2015) 793-801.

[86] N.V. Hud, Nucleic acid-metal ion interactions, RSC Pub., Cambridge, UK, 2009.

[87] M. Steiner, D. Rueda, R.K.O. Sigel, Angew. Chem. 121 (2009) 9920-9924.

[88] M. Helm, A.Y. Kobitski, G.U. Nienhaus, Biophys. Rev. 1 (2009) 161-176.

[89] J.L. Huppert, S. Balasubramanian, Nucleic Acids Res. 35 (2007) 406-413.

[90] O. Kikin, Z. Zappala, L. D'Antonio, P.S. Bagga, Nucleic Acids Res. 36 (2008) D141-8.

[91] J. Deng, Y. Xiong, B. Pan, M. Sundaralingam, Acta Crystallogr. Sect. D 59 (2003) 10041011.

[92] H.R. Drew, R.M. Wing, T. Takano, C. Broka, S. Tanaka, K. Itakura, R.E. Dickerson, Proc. Natl. Acad. Sci. U. S. A. 78 (1981) 2179-2183.

[93] M. Popenda, J. Milecki, R.W. Adamiak, Nucleic Acids Res. 32 (2004) 4044-4054. 
[94] H. Martadinata, A.T. Phan, J. Am. Chem. Soc. 131 (2009) 2570-2578.

[95] K. Snoussi, S. Nonin-Lecomte, J.L. Leroy, J. Mol. Biol. 309 (2001) 139-153.

[96] M. Daher, D. Rueda, Biochemistry 51 (2012) 3531-3538.

[97] J. Ouellet, S. Melcher, A. Iqbal, Y. Ding, Lilley, David M J, RNA 16 (2010) 1597-1609.

[98] Z. Xie, N. Srividya, T.R. Sosnick, T. Pan, N.F. Scherer, Proc. Natl. Acad. Sci. U. S. A. 101 (2004) 534-539.

[99] H.D. Kim, G.U. Nienhaus, T. Ha, J.W. Orr, J.R. Williamson, S. Chu, Proc. Natl. Acad. Sci. U. S. A. 99 (2002) 4284-4289.

[100] T. Ha, X. Zhuang, H.D. Kim, J.W. Orr, J.R. Williamson, S. Chu, Proc. Natl. Acad. Sci. U. S. A. 96 (1999) 9077-9082.

[101] N.G. Walter, C.-Y. Huang, A.J. Manzo, M.A. Sobhy, Nat. Methods 5 (2008) 475-489.

[102] D. Rueda, G. Bokinsky, M.M. Rhodes, M.J. Rust, X. Zhuang, N.G. Walter, Proc. Natl. Acad. Sci. U. S. A. 101 (2004) 10066-10071.

[103] R. Zhao, M. Marshall, E.A. Alemán, R. Lamichhane, A. Feig, D. Rueda, Biophys. J. 99 (2010) 1925-1931.

[104] J.H. Hodak, C.D. Downey, J.L. Fiore, A. Pardi, D.J. Nesbitt, Proc. Natl. Acad. Sci. U. S. A. 102 (2005) 10505-10510.

[105] Pereira, Miguel J B, E.N. Nikolova, S.L. Hiley, D. Jaikaran, R.a. Collins, N.G. Walter, J. Mol. Biol. 382 (2008) 496-509.

[106] B. Okumus, T.J. Wilson, Lilley, David M J, T. Ha, Biophys. J. 87 (2004) 2798-2806.

[107] X. Zhuang, H. Kim, Pereira, Miguel J B, H.P. Babcock, N.G. Walter, S. Chu, Science 296 (2002) 1473-1476.

[108] M.P. Elenko, J.W. Szostak, van Oijen, Antoine M, J. Am. Chem. Soc. 131 (2009) 98669867.

[109] E. Tan, T.J. Wilson, M.K. Nahas, R.M. Clegg, Lilley, David M J, T. Ha, Proc. Natl. Acad. Sci. U. S. A. 100 (2003) 9308-9313.

[110] M.K. Nahas, T.J. Wilson, S. Hohng, K. Jarvie, Lilley, David M J, T. Ha, Nat. Struct. Mol. Biol. 11 (2004) 1107-1113.

[111] N. Toor, K.S. Keating, S.D. Taylor, A.M. Pyle, Science 320 (2008) 77-82.

[112] G. Quigley, A. Rich, Science 194 (1976) 796-806.

[113] E. Ennifar, P. Walter, B. Ehresmann, C. Ehresmann, P. Dumas, Nat. Struct. Biol. 8 (2001) 1064-1068. 
[114] M. Mandal, R.R. Breaker, Nat. Rev. Mol. Cell Biol. 5 (2004) 451-463.

[115] J.E. Johnson, F.E. Reyes, J.T. Polaski, R.T. Batey, Nature 492 (2012) 133-137.

[116] N.F. Dupuis, E.D. Holmstrom, D.J. Nesbitt, Biophys. J. 105 (2013) 756-766.

[117] E.D. Holmstrom, J.T. Polaski, R.T. Batey, D.J. Nesbitt, J. Am. Chem. Soc. 136 (2014) 16832-16843.

[118] N. Salim, R. Lamichhane, R. Zhao, T. Banerjee, J. Philip, D. Rueda, A.L. Feig, Biophys. J. 102 (2012) 1097-1107.

[119] S. Ebbinghaus, A. Dhar, J.D. McDonald, M. Gruebele, Nat. Methods 7 (2010) 319-323.

[120] S. Bernacchi, E. Ennifar, K. Tóth, P. Walter, J. Langowski, P. Dumas, The Journal of biological chemistry 280 (2005) 40112-40121.

[121] K.I. Takahashi, S. Baba, P. Chattopadhyay, Y. Koyanagi, N. Yamamoto, H. Takaku, G. Kawai, RNA (New York, N.Y.) 6 (2000) 96-102.

[122] M.J. Rist, J.P. Marino, Biochemistry 41 (2002) 14762-14770.

[123] H. Mundigala, J.B. Michaux, A.L. Feig, E. Ennifar, D. Rueda, Nucleic Acids Res. 42 (2014) 7281-7289.

[124] R.T. Batey, R.P. Rambo, J.A. Doudna, Angew. Chem. Int. Ed. 38 (1999) 2326-2343.

[125] J.H. Cate, A.R. Gooding, E. Podell, K. Zhou, B.L. Golden, C.E. Kundrot, T.R. Cech, J.A. Doudna, Science 273 (1996) 1678-1685.

[126] J.L. Fiore, J.H. Hodak, O. Piestert, C.D. Downey, D.J. Nesbitt, Biophys. J. 95 (2008) 3892-3905.

[127] P.B. Rupert, A.R. Ferré-D'Amaré, Nature 410 (2001) 780-786.

[128] F. Walter, A.H. Murchie, D.M. Lilley, Biochemistry 37 (1998) 17629-17636.

[129] A. Sigel, H. Sigel (Eds.), Structural and Catalytic Roles of Metal Ions in RNA, Royal Society of Chemistry, Cambridge, 2011.

[130] R.K.O. Sigel, Eur. J. Inorg. Chem. 2005 (2005) 2281-2292.

[131] Y. Wang, M. Xiao, Y. Li, Proteins: Struct., Funct., Bioinf. 82 (2014) 1-9.

[132] N.G. Walter, K.J. Hampel, K.M. Brown, J.M. Burke, EMBO J. 17 (1998) 2378-2391.

[133] G. Bokinsky, D. Rueda, V.K. Misra, M.M. Rhodes, A. Gordus, H.P. Babcock, N.G. Walter, X. Zhuang, Proc. Natl. Acad. Sci. U. S. A. 100 (2003) 9302-9307.

[134] A. Hampel, R. Tritz, Biochemistry 28 (1989) 4929-4933.

[135] D. Kilburn, J.H. Roh, L. Guo, R.M. Briber, S.A. Woodson, J. Am. Chem. Soc. 132 (2010) $8690-8696$. 
[136] S.J. Benkovic, S. Hammes-Schiffer, Science 301 (2003) 1196-1202.

[137] C. de Silva, N.G. Walter, RNA 15 (2009) 76-84.

[138] S.E. McDowell, J.M. Jun, N.G. Walter, RNA 16 (2010) 2414-2426.

[139] S.A. McKinney, C. Joo, T. Ha, Biophys. J. 91 (2006) 1941-1951.

[140] D. Donghi, J. Schnabl, Met. Ions Life Sci. 9 (2011) 197-234.

[141] X. Zhuang, L.E. Bartley, H.P. Babcock, R. Russell, T. Ha, D. Herschlag, S. Chu, Science 288 (2000) 2048-2051.

[142] B.D. Sattin, W. Zhao, K. Travers, S. Chu, D. Herschlag, J. Am. Chem. Soc. 130 (2008) $6085-6087$.

[143] S.K. Silverman, M.L. Deras, S.a. Woodson, S.A. Scaringe, T.R. Cech, Biochemistry 39 (2000) 12465-12475.

[144] R. Russell, X. Zhuang, H.P. Babcock, I.S. Millett, S. Doniach, S. Chu, D. Herschlag, Proc. Natl. Acad. Sci. U. S. A. 99 (2002) 155-160.

[145] G.J. Smith, T.R. Sosnick, N.F. Scherer, T. Pan, RNA 11 (2005) 234-239.

[146] X. Qu, G.J. Smith, K.T. Lee, T.R. Sosnick, T. Pan, N.F. Scherer, Proc. Natl. Acad. Sci. U. S. A. 105 (2008) 6602-6607.

[147] X.W. Fang, B.L. Golden, K. Littrell, V. Shelton, P. Thiyagarajan, T. Pan, T.R. Sosnick, Proc. Natl. Acad. Sci. U. S. A. 98 (2001) 4355-4360.

[148] K.L. Buchmueller, A.E. Webb, D.A. Richardson, K.M. Weeks, Nat. Struct. Biol. 7 (2000) $362-366$.

[149] R. Russell, I.S. Millett, S. Doniach, D. Herschlag, Nat. Struct. Biol. 7 (2000) 367-370.

[150] A.M. Pyle, in: Lilley, David M. J, F. Eckstein (Eds.), Ribozymes and RNA catalysis, Royal Society of Chemistry, Cambridge, 2008, pp. 201-228.

[151] K.S. Karunatilaka, A. Solem, A.M. Pyle, D. Rueda, Nature 467 (2010) 935-939.

[152] M. Erat, R.K.O. Sigel, J. Biol. Inorg. Chem. 13 (2008) 1025-1036.

[153] X. Nou, R.J. Kadner, Proc. Natl. Acad. Sci. U. S. A. 97 (2000) 7190-7195.

[154] S. Gallo, M. Oberhuber, R.K.O. Sigel, B. Kräutler, ChemBioChem 9 (2008) 1408-1414.

[155] E. Loh, O. Dussurget, J. Gripenland, K. Vaitkevicius, T. Tiensuu, P. Mandin, F. Repoila, C. Buchrieser, P. Cossart, J. Johansson, Cell 139 (2009) 770-779.

[156] A. Wachter, M. Tunc-Ozdemir, B.C. Grove, P.J. Green, D.K. Shintani, R.R. Breaker, The Plant cell 19 (2007) 3437-3450.

[157] W.C. Winkler, R.R. Breaker, ChemBioChem 4 (2003) 1024-1032. 
[158] A. Savinov, C.F. Perez, S.M. Block, Biochim. Biophys. Acta 1839 (2014) 1030-1045.

[159] J.-F. Lemay, J.C. Penedo, R. Tremblay, Lilley, David M J, D.A. Lafontaine, Chem. Biol. 13 (2006) 857-868.

[160] J.-F. Lemay, J.C. Penedo, J. Mulhbacher, D.A. Lafontaine, Molecular basis of RNAmediated gene regulation on the adenine riboswitch by single-molecule approaches, Humana Press, Totowa, NJ, 2009.

[161] R.T. Batey, S.D. Gilbert, R.K. Montange, Nature 432 (2004) 411-415.

[162] M.D. Brenner, M.S. Scanlan, M.K. Nahas, T. Ha, S.K. Silverman, Biochemistry 49 (2010) $1596-1605$.

[163] M.F. Soulière, R.B. Altman, V. Schwarz, A. Haller, S.C. Blanchard, R. Micura, Proc. Natl. Acad. Sci. U. S. A. 110 (2013) E3256-64.

[164] A. Haller, U. Rieder, M. Aigner, S.C. Blanchard, R. Micura, Nat. Chem. Biol. 7 (2011) $393-400$.

[165] B. Heppell, S. Blouin, A.-M. Dussault, J. Mulhbacher, E. Ennifar, J.C. Penedo, D.A. Lafontaine, Nat. Chem. Biol. 7 (2011) 384-392.

[166] S. Wood, A.R. Ferré-D'Amaré, D. Rueda, ACS Chem. Biol. 7 (2012) 920-927.

[167] L.R. Fiegland, A.D. Garst, R.T. Batey, D.J. Nesbitt, Biochemistry 51 (2012) 9223-9233.

[168] S. Weiss, Nat. Struct. Biol. 7 (2000) 724-729.

[169] J. Hohlbein, K. Gryte, M. Heilemann, A.N. Kapanidis, Phys. Biol. 7 (2010) 031001.

[170] S. Blouin, T.D. Craggs, D.A. Lafontaine, J.C. Penedo, Functional studies of DNA-protein interactions using FRET techniques, Humana Press, Totowa, NJ, 2009.

[171] Y. Wang, L. Guo, I. Golding, E.C. Cox, N.P. Ong, Biophys. J. 96 (2009) 609-620.

[172] S. Wennmalm, L. Edman, R. Rigler, Proc. Natl. Acad. Sci. U. S. A. 94 (1997) 1064110646.

[173] C.G. Baumann, S.B. Smith, V.A. Bloomfield, C. Bustamante, Proc. Natl. Acad. Sci. U. S. A. 94 (1997) 6185-6190.

[174] J.F. Leger, J. Robert, L. Bourdieu, D. Chatenay, J.F. Marko, Proc. Natl. Acad. Sci. U. S. A. 95 (1998) 12295-12299.

[175] C. Joo, S.A. McKinney, M. Nakamura, I. Rasnik, S. Myong, T. Ha, Cell 126 (2006) 515527.

[176] C.R. Sabanayagam, J.S. Eid, A. Meller, J. Chem. Phys. 122 (2005) 061103.

[177] A. Iqbal, S. Arslan, B. Okumus, T.J. Wilson, G. Giraud, D.G. Norman, T. Ha, Lilley, 
David M J, Proc. Natl. Acad. Sci. U. S. A. 105 (2008) 11176-11181.

[178] P. Schwille, F.J. Meyer-Almes, R. Rigler, Biophys. J. 72 (1997) 1878-1886.

[179] I.H. Stein, C. Steinhauer, P. Tinnefeld, J. Am. Chem. Soc. 133 (2011) 4193-4195.

[180] I.I. Cisse, H. Kim, T. Ha, Nat. Struct. Mol. Biol. 19 (2012) 623-627.

[181] S.L. König, J.L. Huppert, R.K.O. Sigel, A.C. Evans, Nucleic Acids Res. 41 (2013) 74537461.

[182] M. Gellert, M.N. Lipsett, D.R. Davies, Proc. Natl. Acad. Sci. U. S. A. 48 (1962) 20132018.

[183] J.L. Huppert, S. Balasubramanian, Nucleic Acids Res. 33 (2005) 2908-2916.

[184] A.K. Todd, M. Johnston, S. Neidle, Nucleic Acids Res. 33 (2005) 2901-2907.

[185] G.G. Jayaraj, S. Pandey, V. Scaria, S. Maiti, RNA Biol. 9 (2012) 81-86.

[186] G.N. Parkinson, in: S. Neidle, S. Balasubramanian (Eds.), Quadruplex Nucleic Acids, Royal Society of Chemistry, Cambridge, 2006.

[187] K. Halder, J.S. Hartig, in: A. Sigel, H. Sigel (Eds.), Structural and Catalytic Roles of Metal Ions in RNA, Royal Society of Chemistry, Cambridge, 2011, pp. 125-139.

[188] A. Joachimi, A. Benz, J.S. Hartig, Bioorg. Med. Chem. 17 (2009) 6811-6815.

[189] P.S. Shirude, S. Balasubramanian, Biochimie 90 (2008) 1197-1206.

[190] P.S. Shirude, L. Ying, S. Balasubramanian, Chem. Commun. (2008) 2007-2009.

[191] L. Ying, J.J. Green, H. Li, D. Klenerman, S. Balasubramanian, Proc. Natl. Acad. Sci. U. S. A. 100 (2003) 14629-14634.

[192] A. Risitano, K.R. Fox, Biochemistry 42 (2003) 6507-6513.

[193] J.Y. Lee, B. Okumus, D.S. Kim, T. Ha, Proc. Natl. Acad. Sci. U. S. A. 102 (2005) 1893818943.

[194] J.Y. Lee, J. Yoon, H.W. Kihm, D.S. Kim, Biochemistry 47 (2008) 3389-3396.

[195] S. Neidle, FEBS J. 277 (2010) 1118-1125.

[196] S. Balasubramanian, L.H. Hurley, S. Neidle, Nat. Rev. Drug Disc. 10 (2011) 261-275.

[197] T.A. Brooks, L.H. Hurley, Genes Cancer 1 (2010) 641-649.

[198] P.S. Shirude, B. Okumus, L. Ying, T. Ha, S. Balasubramanian, J. Am. Chem. Soc. 129 (2007) 7484-7485.

[199] B. Okumus, T. Ha, in: P. Baumann (Ed.), G-Quadruplex DNA, Humana Press, Totowa, NJ, 2010, pp. 81-96.

[200] A. Fegan, P.S. Shirude, L. Ying, S. Balasubramanian, Chem. Commun. 46 (2010) 946- 
948.

[201] H. Hwang, N. Buncher, P.L. Opresko, S. Myong, Structure 20 (2012) 1872-1880.

[202] D. Hockemeyer, A.J. Sfeir, J.W. Shay, W.E. Wright, T. de Lange, EMBO J. 24 (2005) $2667-2678$.

[203] F. Wang, E.R. Podell, A.J. Zaug, Y. Yang, P. Baciu, T.R. Cech, M. Lei, Nature 445 (2007) $506-510$.

[204] S.L. König, M. Hadzic, E. Fiorini, R. Börner, D. Kowerko, W.U. Blanckenhorn, R.K.O. Sigel, PLoS One 8 (2013) e84157.

[205] D. Kowerko, M.C. Hadzic, R. Börner, S.L. König, R.K.O. Sigel, Proposal accepted in Chemical Review (2015).

[206] F. Cichos, C. von Borczyskowski, M. Orrit, Curr. Opin. Colloid Interface Sci. 12 (2007) $272-284$.

[207] R. Roy, S. Hohng, T. Ha, Nat. Methods 5 (2008) 507-516.

[208] M. Greenfeld, D.S. Pavlichin, H. Mabuchi, D. Herschlag, PLoS One 7 (2012) e30024.

[209] R. Zhao, D. Rueda, Methods 49 (2009) 112-117.

[210] W.P. Ambrose, P.M. Goodwin, J.P. Nolan, Cytometry 36 (1999) 224-231.

[211] J.C. Crocker, D.G. Grier, J. Colloid Interface Sci. 179 (1996) 298-310.

[212] H. Deschout, F. Cella Zanacchi, M. Mlodzianoski, A. Diaspro, J. Bewersdorf, S.T. Hess, K. Braeckmans, Nat. Methods 11 (2014) 253-266.

[213] M. Böhmer, J. Enderlein, ChemPhysChem 4 (2003) 792-808.

[214] C.A. Schneider, W.S. Rasband, K.W. Eliceiri, Nat. Methods 9 (2012) 671-675.

[215] I.F. Sbalzarini, P. Koumoutsakos, J. Struct. Biol. 151 (2005) 182-195.

[216] J. Schuster, F. Cichos, C. von Borzcyskowski, Eur. Polym. J. 40 (2004) 993-999.

[217] R. Parthasarathy, Nat. Methods 9 (2012) 724-726.

[218] M.J. Rust, M. Bates, X. Zhuang, Nat. Methods 3 (2006) 793-795.

[219] S.J. Holden, S. Uphoff, A.N. Kapanidis, Nat. Methods 8 (2011) 279-280.

[220] S.T. Hess, Girirajan, Thanu P K, M.D. Mason, Biophys. J. 91 (2006) 4258-4272.

[221] S.W. Hell, Nat. Methods 6 (2009) 24-32.

[222] A. Small, S. Stahlheber, Nat. Methods 11 (2014) 267-279.

[223] G.S. Harms, M. Sonnleitner, G.J. Schütz, H.J. Gruber, T. Schmidt, Biophys. J. 77 (1999) 2864-2870.

[224] L. Cognet, G.S. Harms, G.a. Blab, P.H. Lommerse, T. Schmidt, Appl. Phys. Lett. 77 
(2000) 4052.

[225] a. Schob, F. Cichos, J. Schuster, C. von Borczyskowski, Eur. Polym. J. 40 (2004) 10191026.

[226] G. Haran, Chem. Phys. 307 (2004) 137-145.

[227] S.H. Chung, R.a. Kennedy, J. Neurosci. Methods 40 (1991) 71-86.

[228] J.N. Taylor, D.E. Makarov, C.F. Landes, Biophys. J. 98 (2010) 164-173.

[229] J.N. Taylor, C.F. Landes, J. Phys. Chem. B 115 (2011) 1105-1114.

[230] C.F. Landes, A. Rambhadran, J.N. Taylor, F. Salatan, V. Jayaraman, Nat. Chem. Biol. 7 (2011) 168-173.

[231] E. Margeat, A.N. Kapanidis, P. Tinnefeld, Y. Wang, J. Mukhopadhyay, R.H. Ebright, S. Weiss, Biophys. J. 90 (2006) 1419-1431.

[232] J.T. Fourkas, Opt. Lett. 26 (2001) 211-213.

[233] R. Börner, D. Kowerko, S. Krause, C. von Borczyskowski, C.G. Hübner, J. Chem. Phys. 137 (2012) 164202.

[234] F.D. Steffen, R.K.O. Sigel, R. Börner, To be submitted.

[235] S.A. McKinney, A.-C. Déclais, D.M. Lilley, T. Ha, Nat. Struct. Biol. 10 (2003) 93-97.

[236] P. Herman, J.C. Lee, Methods Mol. Biol. 796 (2012) 399-421.

[237] L.E. Baum, T. Petrie, Ann. Math. Statist. 37 (1966) 1554-1563.

[238] M. Blanco, N.G. Walter, in: N.G. Walter (Ed.), Fluorescence based approaches, 1st ed., Elsevier, Acad. Press, Amsterdam [u.a.], 2010, pp. 153-178.

[239] K.A. Henzler-Wildman, V. Thai, M. Lei, M. Ott, M. Wolf-Watz, T. Fenn, E. Pozharski, M.A. Wilson, G.A. Petsko, M. Karplus, C.G. Hübner, D. Kern, Nature 450 (2007) 838-844.

[240] J.E. Bronson, J. Fei, J.M. Hofman, R.L. Gonzalez, C.H. Wiggins, Biophys. J. 97 (2009) 3196-3205.

[241] F. Qin, Biophys. J. 86 (2004) 1488-1501.

[242] F. Qin, L. Li, Biophys. J. 87 (2004) 1657-1671.

[243] M. Pirchi, G. Ziv, I. Riven, S.S. Cohen, N. Zohar, Y. Barak, G. Haran, Nat. Commun. 2 (2011) 493.

[244] H. Yang, X.S. Xie, J. Chem. Phys. 117 (2002) 10965.

[245] L.P. Watkins, H. Yang, J. Phys. Chem. B 109 (2005) 617-628.

[246] C.S. Xu, H. Kim, H. Yang, C.C. Hayden, J. Am. Chem. Soc. 129 (2007) 11008-11009.

[247] J.F. Beausang, Y.E. Goldman, P.C. Nelson, in: M.L. Johnson, L. Brand (Eds.), Computer 
methods, 1st ed., Academic Press, San Diego, CA, 2011, pp. 431-463.

[248] R. Schmidt, C. Krasselt, C. von Borczyskowski, Chem. Phys. 406 (2012) 9-14.

[249] D.L. Floyd, S.C. Harrison, Oijen, Antoine M. van, Biophys. J. 99 (2010) 360-366.

[250] C. Hellriegel, J. Kirstein, C. Braeuchle, V. Latour, T. Pigot, R. Olivier, S. Lacombe, R. Brown, V. Guieu, C. Payrastre, A. Izquierdo, P. Mocho, C. Bräuchle, C. Bra, J. Phys. Chem. B 108 (2004) 14699-14709.

[251] S. Myong, M.M. Bruno, A.M. Pyle, T. Ha, Science 317 (2007) 513-516.

[252] J.P. Torella, S.J. Holden, Y. Santoso, J. Hohlbein, A.N. Kapanidis, Biophys. J. 100 (2011) $1568-1577$.

[253] Y. Santoso, J.P. Torella, A.N. Kapanidis, ChemPhysChem 11 (2010) 2209-2219.

[254] S. Farooq, J. Hohlbein, Phys. Chem. Chem. Phys. 17 (2015) 27862-27872.

[255] S. Kalinin, T. Peulen, S. Sindbert, P.J. Rothwell, S. Berger, T. Restle, R.S. Goody, H. Gohlke, C.A. Seidel, Nat. Methods 9 (2012) 1218-1225.

[256] L.L. Schrödinger, The PyMOL Molecular Graphics System, Version 1.8, 2015.

[257] G.P. Quinn, M.J. Keough, Experimental design and data analysis for biologists, Cambridge University Press, Cambridge, UK, New York, 2002.

[258] N. Toor, K. Rajashankar, K.S. Keating, A.M. Pyle, Nat. Struct. Mol. Biol. 15 (2008) 12211222.

[259] I.-B. Lee, S.-C. Hong, N.-K. Lee, A. Johner, Biophys. J. 103 (2012) 2492-2501.

[260] V.B. Chu, Y. Bai, J. Lipfert, D. Herschlag, S. Doniach, Curr. Opin. Chem. Biol. 12 (2008) 619-625.

[261] D. Rueda, J. Hsieh, J.J. Day-Storms, C.A. Fierke, N.G. Walter, Biochemistry 44 (2005) $16130-16139$. 


\section{Tables}

Table 1. Non-comprehensive overview of DNA/RNA molecules investigated by smFRET with regard to metal ion interactions.

\begin{tabular}{|c|c|c|c|}
\hline RNA & $\mathrm{Mg}^{2+}$ ions & other parameters & ref. \\
\hline \multicolumn{4}{|l|}{ Model systems } \\
\hline \multirow[t]{2}{*}{ Three-helix (way) junction } & $1 \mu \mathrm{M}-3 \mathrm{mM}$ & $0-1 \mathrm{M} \mathrm{Na}^{+}$, Protein S15 & {$[8]$} \\
\hline & $0-400 \mu \mathrm{M}$ & $<50 \mathrm{mM} \mathrm{Na}^{+}$ & [99] \\
\hline Four-way junction & $0.1-50 \mathrm{mM}$ & $50 \mathrm{mM} \mathrm{Na}^{+}$ & [109] \\
\hline Kissing complex/loop interaction & $10 \mathrm{mM}$ & $20 \mathrm{mM} \mathrm{Ca}^{2+}, 150 \mathrm{mM} \mathrm{Na}^{+}$ & {$[103,118,261]$} \\
\hline GAAA tetraloop receptor & $0-10 \mathrm{mM}$ & $100 \mathrm{mM} \mathrm{Na}^{+}$ & {$[104]$} \\
\hline Holliday junction & $0,0.5,50 \mathrm{mM}$ & $50 \mathrm{mM} \mathrm{Na}^{+}$ & {$[21]$} \\
\hline \multicolumn{4}{|l|}{ Ribozymes } \\
\hline \multirow[t]{3}{*}{ Hairpin ribozyme } & $0-500 \mathrm{mM}$ & various $\left[\mathrm{Na}^{+}\right]$ & {$[133]$} \\
\hline & $0-10 \mathrm{mM}$ & $50 \mathrm{mM} \mathrm{Na}^{+}$ & {$[110]$} \\
\hline & $12 \mathrm{mM}$ & $50 \mathrm{mM} \mathrm{Na}^{+}$ & {$[107]$} \\
\hline Group I intron (T. thermophila) & $10-300 \mathrm{mM}$ & $200 \mathrm{mM} \mathrm{Na}^{+}$ & {$[142]$} \\
\hline \multirow[t]{3}{*}{ Group IIB intron (yeast, S. cerevisiae) } & $0-100 \mathrm{mM}$ & $500 \mathrm{mM} \mathrm{K}^{+}$ & {$[75]$} \\
\hline & $1-100 \mathrm{mM}$ & $500 \mathrm{mM} \mathrm{K}^{+}, 1-10 \mathrm{mM} \mathrm{Ca}^{2+}$ & {$[22]$} \\
\hline & $8 \mathrm{mM}$ & $100 / 500 \mathrm{mM} \mathrm{K}^{+}, \mathrm{Mss} 116$ & {$[151]$} \\
\hline Varkud satellite (VS) ribozyme & $35 \mathrm{mM}$ & $50 \mathrm{mM} \mathrm{K}^{+}$ & {$[105]$} \\
\hline Hammerhead ribozyme & $0-10 \mathrm{mM}$ & $0 / 100 \mathrm{mM} \mathrm{K}^{+}$ & [138] \\
\hline \multicolumn{4}{|l|}{ Riboswitches/Miscellaneous } \\
\hline c-di-GMP-I riboswitch & $0-25 \mathrm{mM}$ & $\begin{array}{l}50 \mathrm{mM} \mathrm{K}^{+}, 50 \mathrm{mM} \mathrm{Na}^{+}, \\
\text {c-di GMP }\end{array}$ & {$[166]$} \\
\hline Lysine riboswitch & $1 / 10 \mathrm{mM}$ & $50 \mathrm{mM} \mathrm{K}^{+}, 100 \mathrm{mM} \mathrm{Na}$ & {$[167]$} \\
\hline RNAse P (Bacillus Subt. \& Stear.) & $0-10 \mathrm{mM}$ & $50 \mathrm{mM} \mathrm{Na}^{+}$ & {$[98,146]$} \\
\hline env8 hydroxocobalamin riboswitch & $0-100 \mathrm{mM}$ & $\begin{array}{l}125 \mathrm{mM} \mathrm{K}, 10 \mathrm{mM} \mathrm{Na}^{+} \text {, } \\
0-1 \mu \mathrm{M}[\mathrm{HyCbl}]\end{array}$ & {$[117]$} \\
\hline \multicolumn{4}{|l|}{ DNA/ G-Quadruplexes (GQ) } \\
\hline GQ: telomeric sequence (htelo) & & $0 / 10 / 100 \mathrm{mM} \mathrm{Na}^{+}$and $\mathrm{K}^{+}$ & [191] \\
\hline GQ: Oxytricha nova telomeric DNA & & $\begin{array}{l}0.1-1000 \mathrm{mM} \mathrm{Na} / \mathrm{K}^{+}, 0.01-1 \\
\mathrm{M} \mathrm{Li}^{+}\end{array}$ & {$[194]$} \\
\hline
\end{tabular}


GQ: human c-kit/c-myc promoter

$0 / 100 \mathrm{mM} \mathrm{K}^{+}$

$[190,198]$ 


\section{Figures}
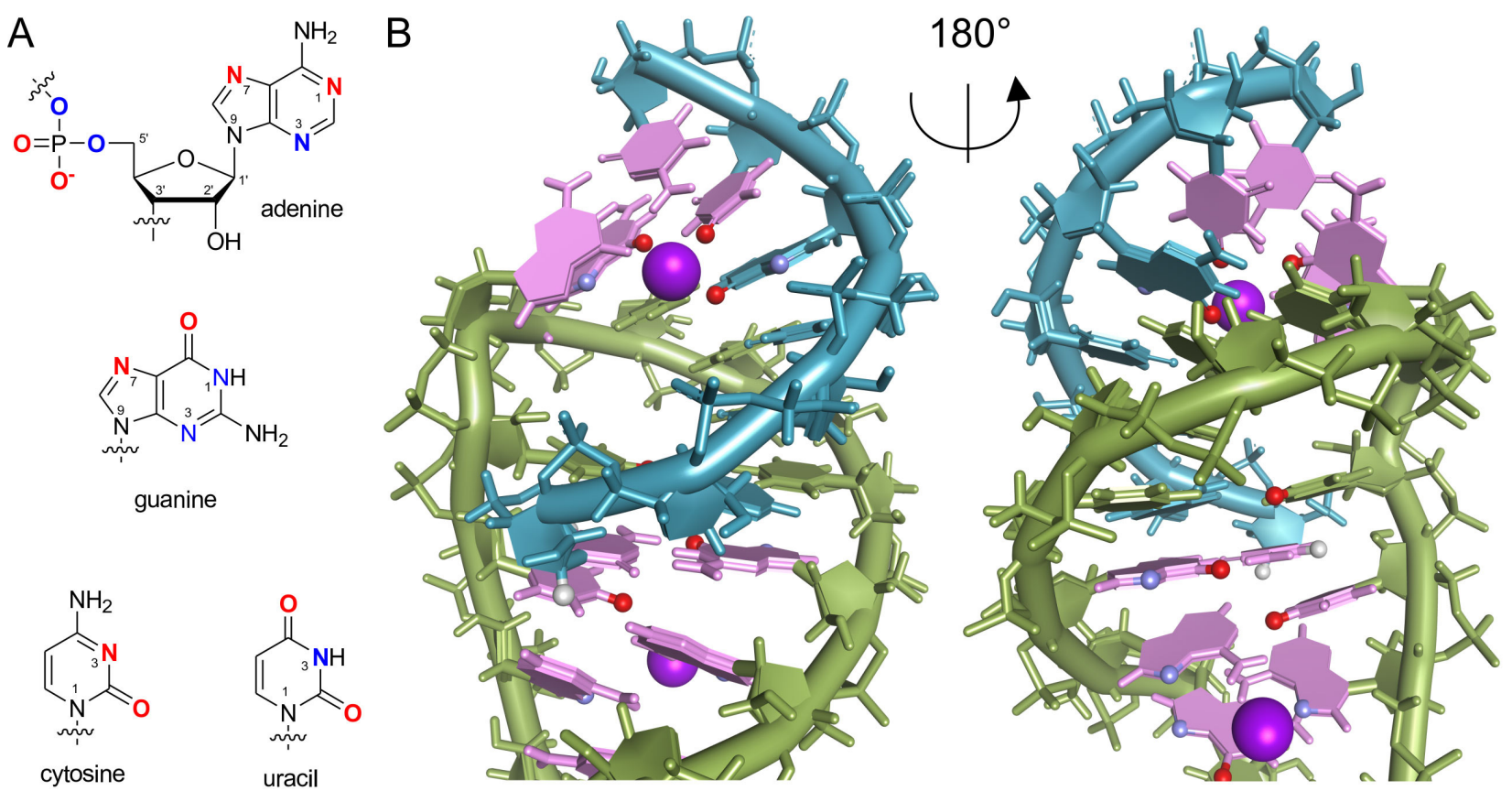

Figure 1. A) Structure of the RNA backbone and the four most abundant nucleobases. The nucleotides contain several donor atoms each for metal-ion coordination, as indicated in red. In addition, the bridging oxygen atoms of the phosphates, the $\mathrm{N} 3$ position of purines, as well as deprotonated guanine $\mathrm{N}^{-}$and uracil $\mathrm{N}^{-}$can also act as coordination sites, as indicated in blue. Panel adapted from [49]. B) Metal ion coordination and charge compensation illustrated for the 5'splice site formation of $\mathrm{d} 3{ }^{\prime} \mathrm{EBS} 1 *$ (green) and IBS $1 *$ (turquoise) (loop-loop interaction). Potential binding sites (involved nucleotides in purple) for $\mathrm{Mg}^{2+}$ (violet) coordination determined by NMR chemical shift change assays [50]: A first binding site is located between A10, U11, and A20 being the first two and the last nucleotide of the loop, and the U9•G21 wobble pair closing the loop of d3'EBS1* (bottom) as well as C59 corresponding to the 5'-end of IBS1*. A second binding site is located between the two first base pairs of the splice site G13•C65 and G14•U64 (3'-end of IBS1*). Each binding site can be occupied by both coordination types. However, there is evidence that the $\mathrm{Mg}^{2+}$ ion close to the stem of $\mathrm{d}^{\prime} \mathrm{EBS}^{*}$ is partially dehydrated, i.e. inner sphere coordinated. Oxygen and nitrogen atoms of the bases able to attract $\mathrm{Mg}^{2+}$ are shown in red and blue. H1' and $\mathrm{H} 5$ protons (grey) are strongly affected upon $\mathrm{Mg}^{2+}$ ion binding in nucleotide $\mathrm{C} 59$ of 
IBS1*. The nucleotide labelling is in accordance with PDB entry 2M23 [50], the structure is generated with the Discovery Studio Visualizer [51]. 


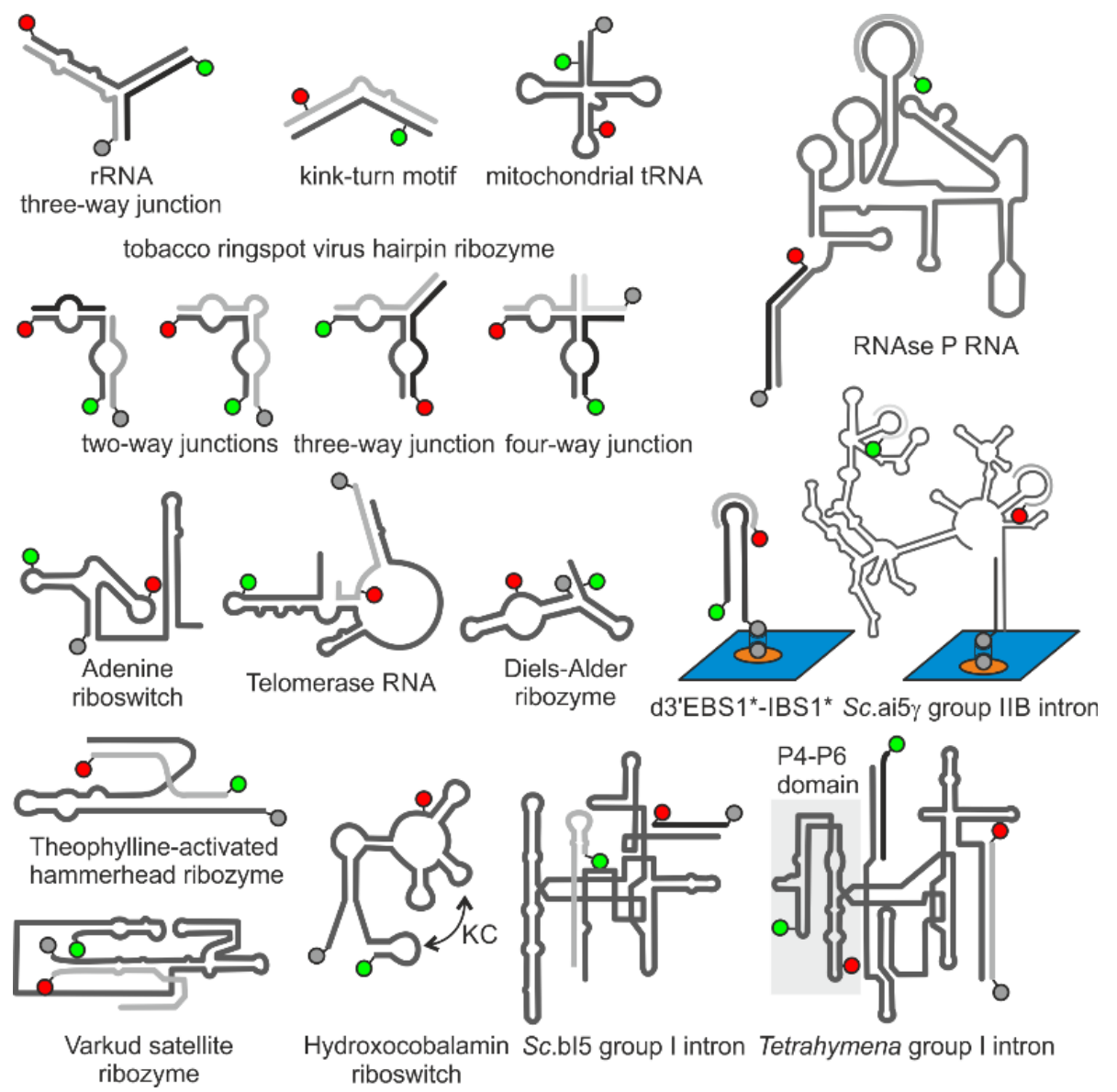

Figure 2. Non-exhaustive scheme of RNA molecules studied using smFRET. Green and red spheres indicate the position of the FRET pair, the donor and the acceptor fluorophore, respectively. When applicable, grey spheres represent biotin moieties for surface immobilization ( $3^{\text {rd }}$ row). The $5^{\prime}$-splice site formation (EBS1-IBS1 interaction) and the group IIB intron of $S$. Cerevisiae are depicted immobilized via biotin-streptavidin binding on a biotin-BSA passivated glass/quartz surface. Figure adapted from [88]. 

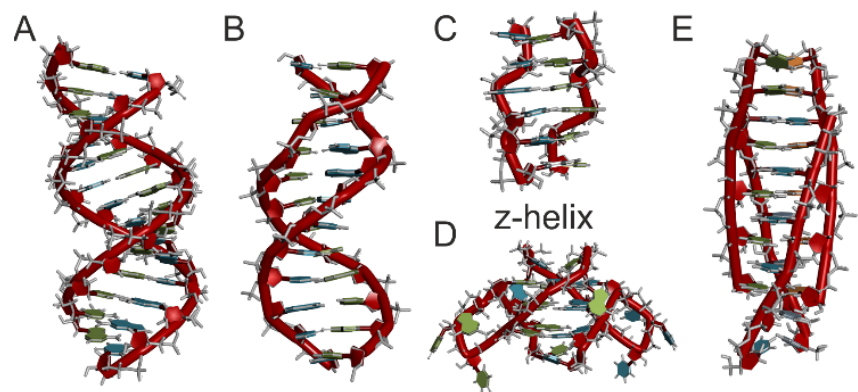

A-helix

B-helix

G-quadruplex

i-motif

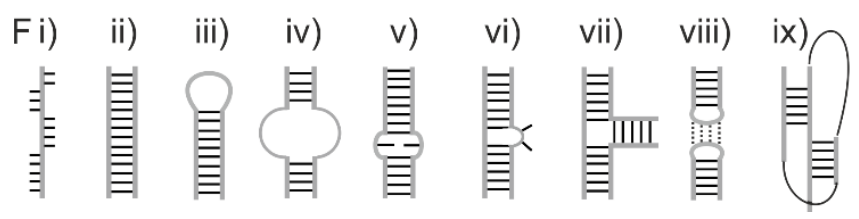

Figure 3. A) The A-form double helix is predominantly found in RNA whereas B) the B-helix of DNA is not adopted by RNA. C) RNA may form the Z-form helix under certain conditions. Fourstranded structures formed by RNA include D) G-quadruplexes, and E) i-motifs. Structures are taken from the PDB Bank, entries 1LNT [91], 1BNA [92], 1T4X [93], 2KBP [94], and 1I9K [95] and the structures are generated with the Discovery Studio Visualizer [51]. F) Examples of RNA secondary and tertiary structure motifs: i) single strand, ii) double helix, iii) hairpin, iv) internal loop, v) mismatch, vi) bulge, and vii) 3-way junction. Tertiary structure motifs found in RNA are viii) kissing loop/complex interaction, and ix) pseudoknot. 


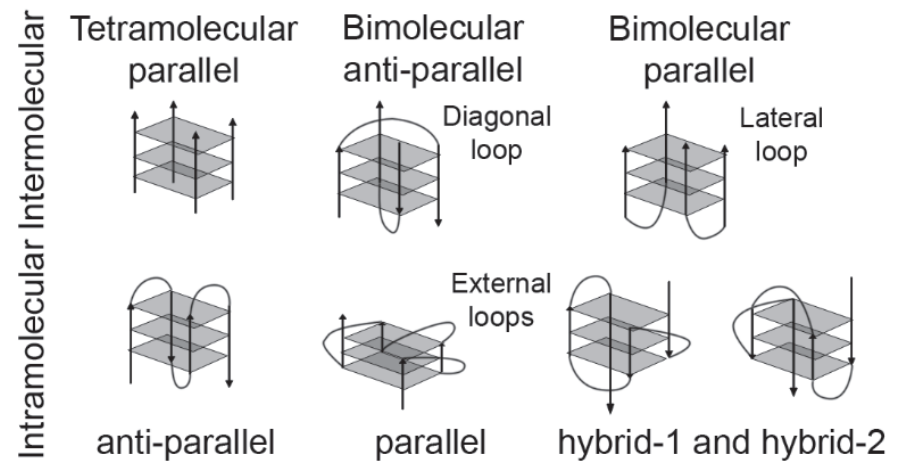

Figure 4. Possible topologies of GQs, here with three consecutive G tetrades depicted as parallel planes: tetramolecular, bimolecular or intramolecular; parallel-stranded, antiparallel-stranded or hybrids of both. The resulting diverse loop topologies are also shown: lateral loops, diagonal loops (requiring two or more nucleotides) or external loops (also called external chain reversal, typical of parallel motifs). Less common topologies are omitted for clarity. 


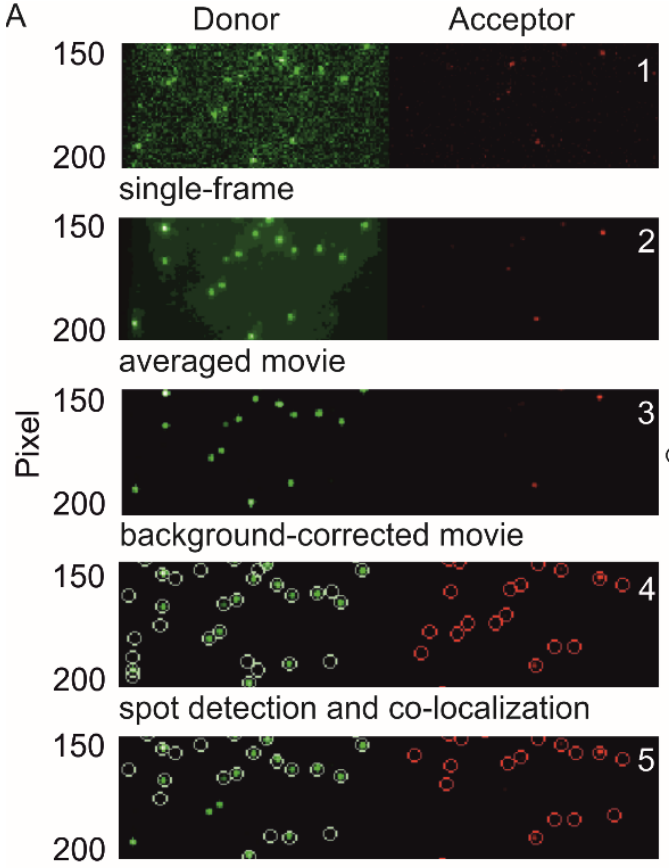

spot exclusion single spot

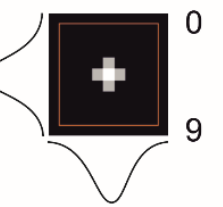

two spots

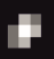
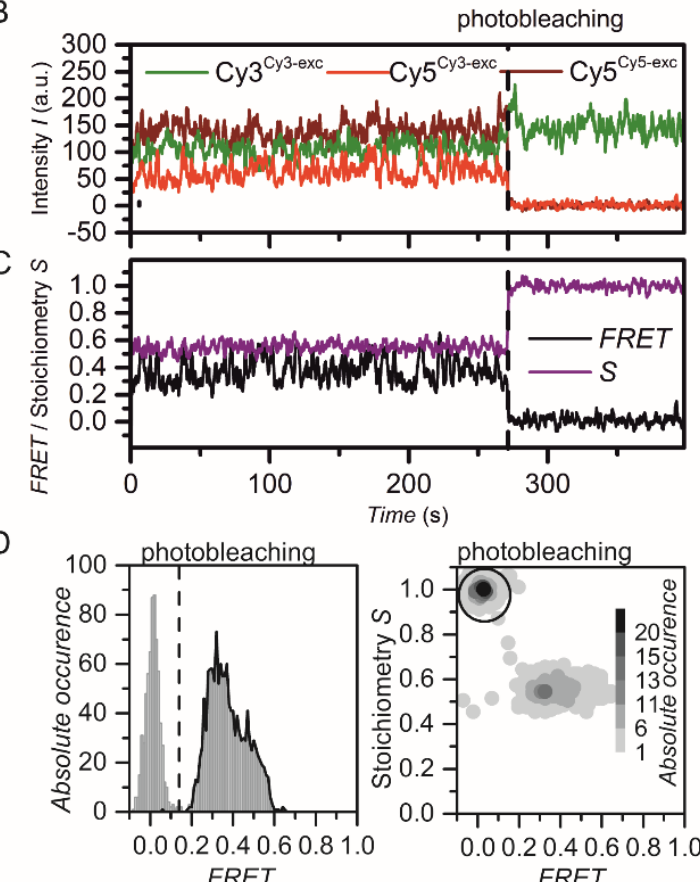

Figure 5. smFRET movie analysis with the software package MASH [205] illustrated with the help of a folding experiment of the FRET construct D135L14 of the group IIB intron labelled with the FRET pair $\mathrm{Cy} 3 / \mathrm{Cy} 5$ at a $\mathrm{Mg}^{2+}$ concentrations of $100 \mathrm{mM}$ (see depiction in Figure 1 and Figure 6 for further exp. details). A) Two-channel movie processing to extract single-molecule intensity-time traces: (from top to bottom) (1) single frame and (2) average image of all frames showing background, (3) average image after applying a Crocker-Grier filter to erase background, (4) channel independent sm localization e.g. with the help of a Houghpeaks algorithm (targeting one bright pixel by a $5 \times 5$ pixel restricted area), and (5) co-localizing associated spots in the donor and acceptor channel, e.g. with a local mean weight spatial transformation, as well as removing spots closer than 4 pixel apart from each other. B) The generated and corrected intensity-time traces of the donor in green and the acceptor in red after green excitation. The acceptor is shown in dark red after direct excitation (ALEX). All intensity traces are denoised with the non-linear Haran filter. C) The calculated apparent FRET as well as the Stoichiometry $S$ time traces are plotted in black and violet. D) The respective FRET histogram (with and without photobleached part of the time trace in C) is plotted together with the respective FRET-S density plot. [Unpublished data] 

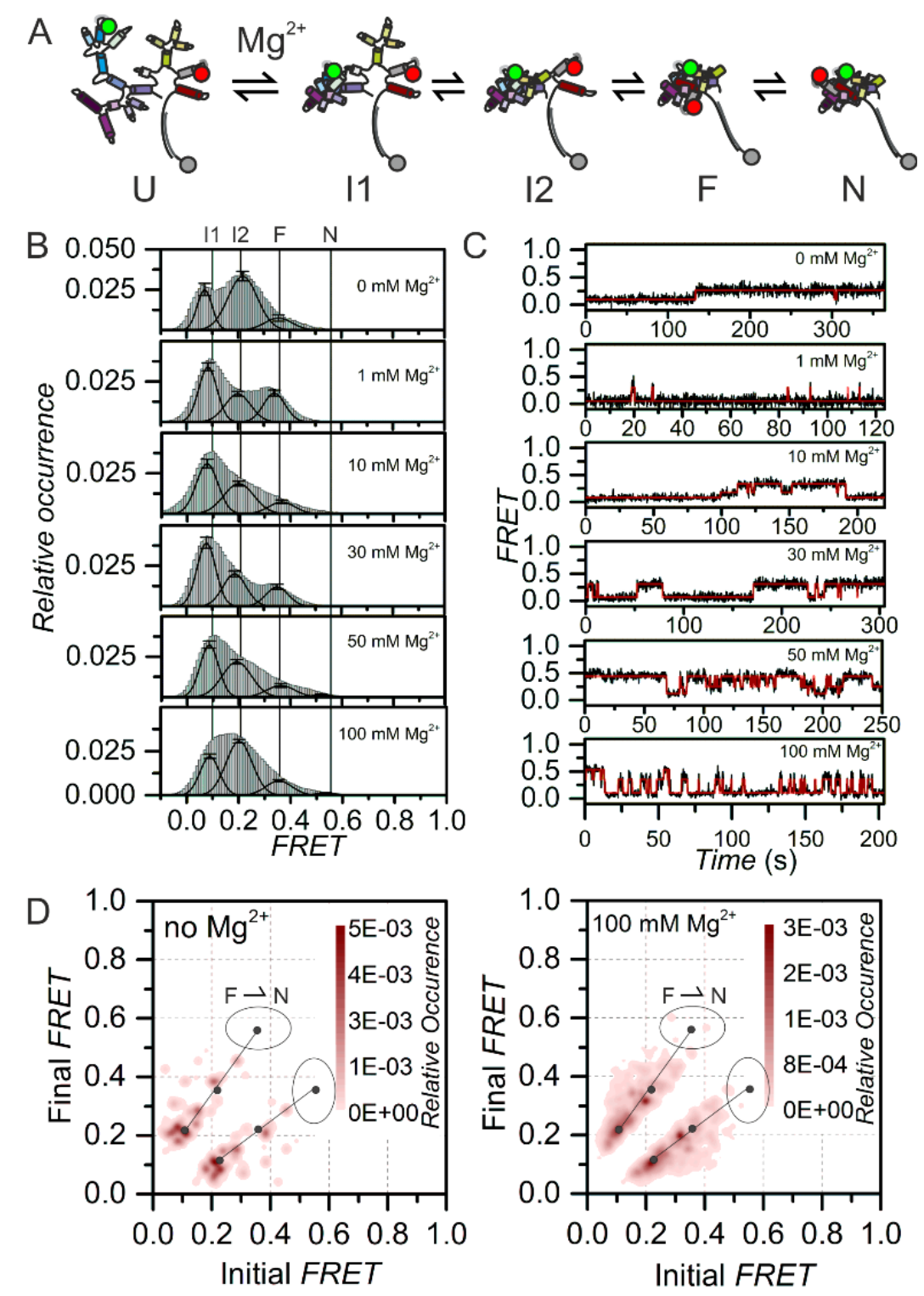

Figure 6. A) Suggested folding pathway of the group IIB intron derivative D135L14 of $S$.

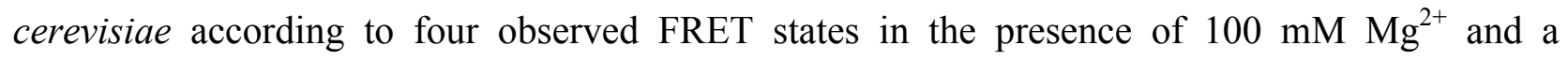
background of $500 \mathrm{mM} \mathrm{K}^{+}$in vitro [76]. B) FRET histogram of a $\mathrm{Mg}^{2+}$ titration (0 to $100 \mathrm{mM}$ $\left.\mathrm{Mg}^{2+}\right)$ and the respective Gaussian functions representing up to four FRET states. The bootstrap interval is plotted as error bar. Adapted from [75]. C) smFRET time traces for increasing $\mathrm{Mg}^{2+}$ concentrations are plotted together with the HMM based discretized dwell times on the right. D) The respective TDPs for two different $\mathrm{Mg}^{2+}$ concentrations (0 and $\left.100 \mathrm{mM}\right)$ show on-axis and sparsely populated off-axis transitions. The latter are visible above or below the indicated direct transitions between neighboring states and might be due to the limited time resolution (100ms). 
The rarely occurring, transient native state transitions of D135L14 are highlighted as black, circled clusters 

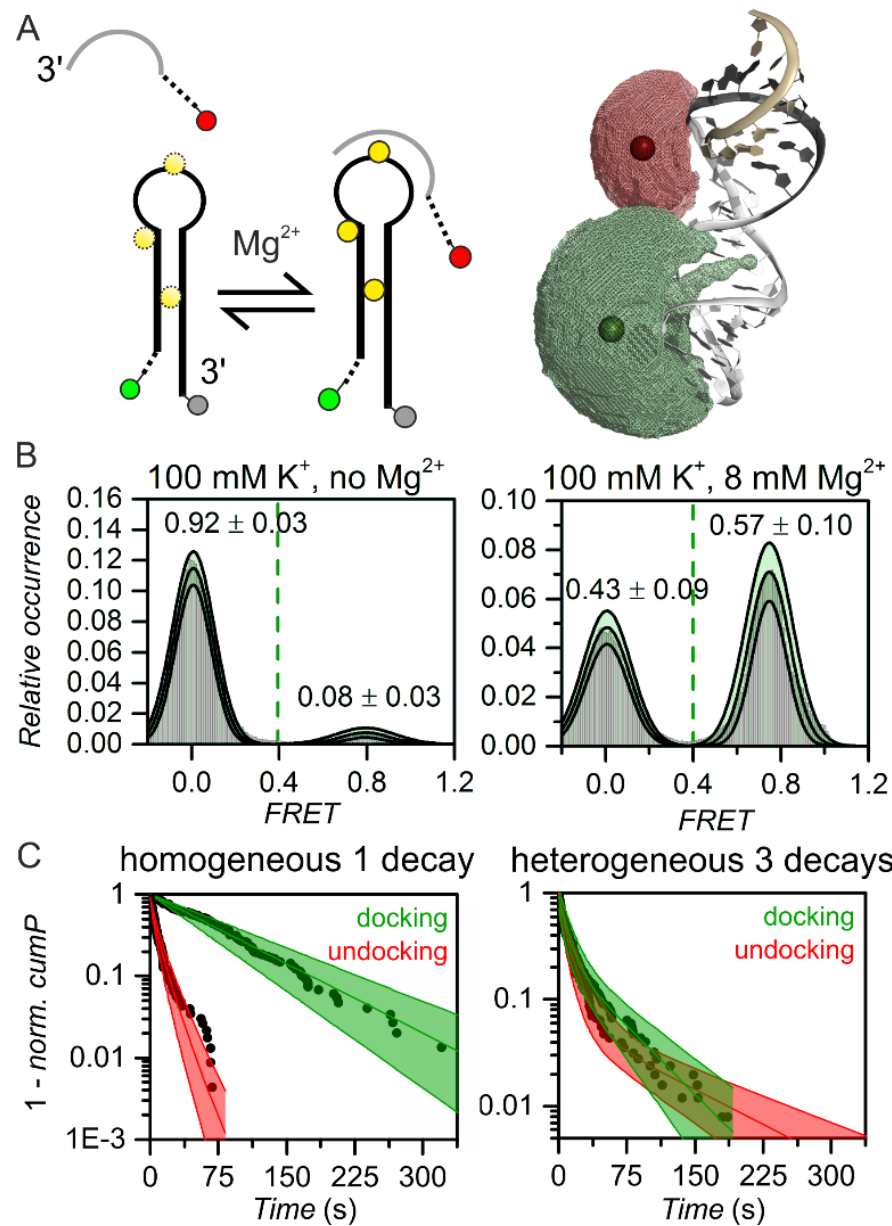

Figure 7. 5'-splice site formation of EBS1*-IBS1* upon $\mathrm{Mg}^{2+}$ binding. A) Equilibrium scheme of the EBS1*-IBS1* interaction upon addition of $\mathrm{Mg}^{2+}$. Predicted $\mathrm{Mg}^{2+}$ binding sites are shown as yellow spheres [50]. Labelling with Cy3 (green spheres) and Cy5 (red spheres) as FRET pair is further depicted on the right along with an accessible volume calculation [255] of the two fluorophores [unpublished data]. The accessible volumes of the respective fluorophores are shown as green and red areas. Based on PDB entry 2M23 [50] the structure was prepared using PyMOL [256]. B) Bootstrapped FRET histogram and C) dwell-time histogram to exemplify the sample variability in absence and presence of $\mathrm{Mg}^{2+}$. Bootstrapping: $M=100$ replicates and $N=n$ of at least $n=90$ molecules for each condition. Adapted from [74,204]. 